\title{
Qualitative and quantitative investigation of the propagation noise in various reactor systems
}

\author{
V. Dykin, A. Jonsson and I. Pázsit \\ Chalmers University of Technology, Department of Nuclear Engineering, \\ SE-412 96 Göteborg, Sweden
}

\begin{abstract}
The space-dependent neutron noise, induced by propagating perturbations (propagation noise for short) is investigated in a one-dimensional homogeneous model of various reactor systems. By using two-group theory, the noise in both the fast and the thermal group is calculated. The purpose is to investigate the dependence of the properties of the space-dependent fast and thermal propagation noise on the static neutron spectrum as well as on the presence of the fluctuations of several cross sections. The motivation for this study arose in connection with recent work on neutron noise in molten salt reactors (MSR) with propagating fuel of various compositions. Some new features of the induced noise were observed, but it was not clear whether these were due to the propagating perturbation alone, or to the propagation of the fuel and hence that of the delayed neutron precursors. The present study serves to clarify the significance of the spectral properties of the different cores through calculating the propagation noise in four different reactor systems, as well as considering the influence of the perturbation of the various cross sections. By comparing the results with those obtained in MSR, the effect of the moving fuel on the propagation noise is clarified. It is shown that in fast systems the noise in the fast group is much larger than in the thermal group and hence can gain diagnostic importance. It is also shown that the co-existence of several cross section fluctuations leads to qualitatively and quantitatively new characteristics of the noise, hence it is important to model the effect of e.g. temperature fluctuations of the coolant in a proper way.
\end{abstract}

\section{Introduction}

The space-dependent behaviour of the neutron noise in power reactors was investigated intensively in a variety of papers for the last 40 years (for an overview, see (Williams, 1974; Kosály, 1980; Pázsit and Demazière, 2010)). 
Somewhat remarkably, however, as noted recently (Pázsit and Dykin, 2010), the in-core noise in PWRs and BWRs, induced by propagating perturbations (inlet temperature fluctuations and two-phase flow, respectively), has remained an exception. The reasons for this are also discussed in Pázsit and Dykin (2010). The space dependence here refers to the axial dependence of the induced noise, i.e. its space dependence in the same direction as the flow/propagation itself. The radial dependence of the neutron noise induced by a local (channel-type) instability was investigated in the past; however, in this case, the axial structure and hence the propagating character of the noise source does not play a role.

The space dependence of the neutron noise induced by propagating perturbations (for which the shorthand notation "propagation noise" will be used further in this paper) received interest recently due to investigations of the neutron noise in molten salt reactors (MSR). In such reactors the circulation of the fuel is likely to induce propagating perturbations due to inhomogeneities in the density/temperature, fuel concentration in the salt, locally slightly varying burnup in the fuel etc. The propagation noise induced by such perturbations was investigated in some recent publications (Pázsit and Jonsson, 2010; Jonsson and Pázsit, 2011).

In the second of the above references the neutron noise was calculated in a two-group approach, in three different systems with different material properties which lead to different neutron spectra. The motivation came from the fact that the MSR, being one of the six selected Gen-IV systems, can be built either with a fast or with a thermal spectrum, dependent on the moderator and the fuel used. Hence three different systems were selected, one with a very soft thermal spectrum, representing a graphite moderated thorium fuelled MSR, a Gen-II light water reactor (BWR) and a high conversion fast system operating with MOX fuel, with fast spectrum. Even these two latter systems were assumed to contain moving fuel in liquid form, representing models of possible MSRs, only the group constants were taken from the corresponding traditional systems. By selecting these three systems, the significance of the spectral properties of the core on the spatial and frequency properties of the induced propagation noise, including the relative significance of the local component of the noise, could be investigated.

The above mentioned investigation was the first of its kind because the spectral properties of the neutron noise, and the significance of the spectral properties of the core on the characteristics of the neutron noise have not been investigated before. All quantitative studies were made in thermal LWRs, and only the influence of the system size and the frequency on the thermal neutron noise was of interest. The investigation of the characteristics of the noise in systems with different spectral properties yielded some new features and tendencies regarding the induced noise. However, it was a somewhat unfortunate 
circumstance that these novel studies were not performed in traditional systems with stationary fuel, rather directly on MSR-type systems with moving fuel, i.e. with moving delayed neutron precursors. Hence it was not possible to say whether these new properties were due to the effect of the propagating perturbations and/or the spectral properties alone, or to the propagation of the fuel and hence that of the delayed neutron precursors. In other words, it was not clear if the same properties would be observed in traditional systems with stationary fuel.

Hence we decided to make a thorough investigation of the noise properties in various traditional systems (i.e. with stationary fuel) with widely differing spectral properties. Four different systems were selected:

- a fast reactor with MOX fuel

- a BWR

- a PWR

- a heavy water core (representing a CANDU).

By selecting these four different systems, a wider range of spectral properties could be covered. This way the present study can also be considered as the first attempt to study the applicability of noise analysis to systems significantly different from the LWR cores dominating in quantitative studies of noise diagnostics so far, as well as the first attempt to study the spectral properties of the induced neutron noise in a two-group model. The present study also serves to clarify the significance of the core properties and that of the fuel propagation, through calculating the propagation noise in cores with static fuel in the four different reactor systems ranging from Gen-II to GEN-IV characteristics and comparing it with the results obtained in the MSR study of Jonsson and Pázsit (2011). One result of these investigations is that in fast systems the neutron noise in the fast group (the "fast noise") has a much larger amplitude than that of the thermal noise, and hence it can be utilised in fast system for diagnostic purposes.

Another novelty of the present investigations is the modelling of the noise source. In the past studies of the propagation noise, the perturbation was modelled by the fluctuations of one single macroscopic cross section:either the thermal absorption cross section (in case of inlet coolant temperature fluctuations in PWRs), or the removal cross section (for the void fluctuations in BWRs). This is because it was considered that the main features of the problem can be described sufficiently well by considering the fluctuations of a "dominating" cross section. The validity of this assumption is investigated in this paper by considering the noise source as temperature fluctuations of the coolant, and calculating the relative weight of the fluctuations of all cross sections. It turned out that the effect of the aggregate of the cross section fluctuations has qualitatively and quantitatively different properties than in 
the case of a single cross section fluctuation. This underlines the need for proper modelling of noise sources at least for calculating the neutron noise induced by propagating temperature and density fluctuations.

\section{Basic considerations}

The investigation of the neutron noise induced by various perturbations is usually performed through the Green's function method. This has the practical advantage that it is more straightforward to obtain analytical results, as well as that the Green's function itself, representing the dynamic transfer function of the system, gives insight into the properties of the system which are not dependent on the perturbation. Hence in the final result it is easier to identify if a certain feature can be attributed to the system properties or to the perturbation.

In a two-group approach, there is an alternative possibility due to the fact that the two-group equations are not self-adjoint. Hence, as was suggested by van Dam $(1975,1976)$, one can use the dynamic adjoint function instead of the Green's function (Pázsit and Demazière, 2010). Actually, due to some differences between the direct and the adjoint Green's function, in most works the dynamic adjoint was used. One reason is that in the two-group case, where both the noise source and the induced neutron noise appear both in the fast and the thermal group, the transfer function is actually a $2 \times 2$ matrix. Although the noise source appears in both groups, in traditional systems one only measures the thermal noise, and hence the calculations were also aimed at only this quantity. In that case, the adjoint approach has the advantage over the direct Green's function that it is sufficient to use the second column of the adjoint matrix, which can be determined from a vector equation. This two-component quantity was called the adjoint function, with a fast and a thermal component, similarly to the flux. Using the direct Green's matrix, to calculate the thermal noise induced by perturbations in both the fast and the thermal group, one would need the second row of the Green's matrix, which cannot be determined from one single vector equation, rather the whole Green's matrix needs to be calculated.

In the present study, given the fact that fast systems will also be investigated, it will be interesting to calculate not only the thermal noise (neutrons with energies below $1 \mathrm{eV}$ ), but also the fast noise (neutrons with energies above $1 \mathrm{eV}$ ). Indeed, in Gen-IV systems with a fast spectrum, it may be either advantageous, or simply necessary, to use the fast noise, or both the fast and the thermal noise, for maximum performance. In that case the advantage of the dynamic adjoint disappears. Hence in the paper both the adjoint function (adjoint matrix) and the Green's matrix will be used, and in all cases both 
the fast and the thermal neutron noise will be calculated and compared.

The formulae used are as follows. The static equations for the direct flux read as:

$$
\left(\begin{array}{cc}
D_{1} \nabla^{2}-\Sigma_{a 1}-\Sigma_{R}+\nu \Sigma_{f 1} & \nu \Sigma_{f 2} \\
\Sigma_{R} & D_{2} \nabla^{2}-\Sigma_{a 2}
\end{array}\right)\left[\begin{array}{l}
\phi_{1}(z) \\
\phi_{2}(z)
\end{array}\right]=0,
$$

where all symbols have their usual meaning. In the above it was assumed that the system is critical, i.e. $k_{e f f}=1$. The notation $\Sigma_{1}=\Sigma_{a 1}+\Sigma_{R}-\nu \Sigma_{f 1}$ will also be employed. Zero flux conditions will be used at the extrapolated boundaries, i.e. $\phi_{i}(0)=\phi_{i}(H)=0$ for a one-dimensional system lying between $z=0$ and $z=H$.

The static fluxes are given as:

$$
\begin{gathered}
\phi_{1}(z)=\sin B_{0} z, \\
\phi_{2}(z)=\frac{\Sigma_{R}}{\Sigma_{a 2}+D_{2} B_{0}^{2}} \sin B_{0} z
\end{gathered}
$$

with $B_{0}=\pi / H$. Here the normalisation was used that the maximum amplitude value of the fast flux is unity. As is known, the equations for the static adjoints $\phi_{1,2}^{\dagger}(z)$ are simply obtained by transposing the matrix on the l.h.s. of (1), and are not written out explicitly.

The equation for the direct Green's matrix is obtained by first turning to the noise equations with the usual procedure of linearisation and temporal Fourier transform. The equation for the group noises reads as:

$$
\left(\begin{array}{cc}
D_{1} \nabla^{2}-\Sigma_{1}(\omega) & \nu \Sigma_{f 2}(\omega) \\
\Sigma_{R} & D_{2} \nabla^{2}-\Sigma_{2}(\omega)
\end{array}\right)\left(\begin{array}{c}
\delta \phi_{1}(z, \omega) \\
\delta \phi_{2}(z, \omega)
\end{array}\right)=\left(\begin{array}{c}
S_{1}(z, \omega) \\
S_{2}(z, \omega)
\end{array}\right) .
$$

Here the noise sources $S_{1}$ and $S_{2}$ are defined in terms of the cross section fluctuations and the static flux in the form:

$$
\begin{aligned}
S_{1}(z, \omega)= & \left(\delta \Sigma_{R}(z, \omega)+\delta \Sigma_{a_{1}}(z, \omega)-\delta \Sigma_{f_{1}}(z, \omega)\left(1-\frac{i \omega \beta}{i \omega+\lambda}\right)\right) \phi_{1}(z)- \\
& \delta \Sigma_{f_{2}}(z, \omega)\left(1-\frac{i \omega \beta}{i \omega+\lambda}\right) \phi_{2}(z)
\end{aligned}
$$

and

$$
S_{2}(z, \omega)=-\delta \Sigma_{R}(z, \omega) \phi_{1}(z)+\delta \Sigma_{a_{2}}(z, \omega) \phi_{2}(z) .
$$

For reasons of simplicity, in the present study the fluctuations of the diffusion coefficients were neglected. This is in accordance with standard practice, and is justified by the fact that the effect of the fluctuations of the diffusion coefficients is much smaller than that of the other cross sections (Pázsit, 2002)). 
The Green's matrix that connects the noise in the fast and thermal group with the fast and thermal noise sources can then be written as:

$$
\begin{aligned}
&\left(\begin{array}{cc}
D_{1} \nabla^{2}-\Sigma_{1}(\omega) & \nu \Sigma_{f 2}(\omega) \\
\Sigma_{R} & D_{2} \nabla^{2}-\Sigma_{2}(\omega)
\end{array}\right)\left(\begin{array}{cc}
G_{11}\left(z, z^{\prime}, \omega\right) & G_{12}\left(z, z^{\prime}, \omega\right) \\
G_{21}\left(z, z^{\prime}, \omega\right) & G_{22}\left(z, z^{\prime}, \omega\right)
\end{array}\right)= \\
&\left(\begin{array}{cc}
\delta\left(z-z^{\prime}\right) & 0 \\
0 & \delta\left(z-z^{\prime}\right)
\end{array}\right),
\end{aligned}
$$

where the usual notations $\Sigma_{1}(\omega)=\Sigma_{a 1}+\Sigma_{R}+\frac{i \omega}{v_{1}}-\Sigma_{f 1}(\omega), \Sigma_{2}(\omega)=\Sigma_{a 2}+\frac{i \omega}{v_{2}}$ and $\Sigma_{f i}(\omega)=\Sigma_{f i}\left(1-\beta \frac{i \omega}{\lambda+i \omega}\right)$ were introduced. Eq. (7) can be written in a symbolic notation as:

$$
\hat{\mathbf{L}}(z, \omega) \hat{\mathbf{G}}\left(z, z^{\prime}, \omega\right)=\hat{\mathbf{I}} \delta\left(z-z^{\prime}\right),
$$

where $\hat{\mathbf{I}}$ is the $2 \times 2$ unit matrix. From Eq. (7) the noise can be obtained as:

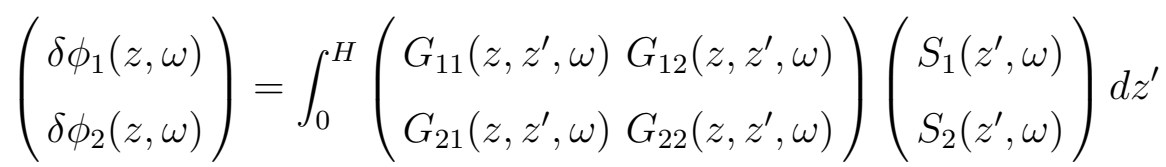

or, again in symbolic form, as:

$$
\delta \vec{\Phi}(z, \omega)=\int \hat{\mathbf{G}}\left(z, z^{\prime}, \omega\right) \overrightarrow{\mathbf{S}}\left(z^{\prime} \omega\right) d z^{\prime}
$$

The elements of the above Green's matrix express the transfer between the noise source and the induced noise in the various groups. Thus, $G_{12}$ connects the effect of a noise source in the thermal group at space point $z^{\prime}$ to the noise induced at point $z$ in the fast group ${ }^{1}$. From here it is seen that for the determination of the thermal noise only, one needs the second row of the Green's matrix. However, this quantity alone cannot be determined from a simpler vector equation, having only two components, rather all four components of the Green's matrix need to be determined simultaneously. In our case, we will be interested in the noise in both the fast and the thermal group, hence we will need the full Green's matrix anyway.

Defining

$$
\begin{gathered}
G_{i}\left(z, z^{\prime}, \omega\right)= \\
=\frac{1}{D_{1} D_{2}\left(\nu^{2}+\mu^{2}\right)} \begin{cases}\frac{\sin \mu\left(H-z^{\prime}\right) \sin \mu z}{\mu \sin \mu H}-\frac{\sinh \nu\left(H-z^{\prime}\right) \sinh \nu z}{\nu \sinh \nu H} & z<z^{\prime}, \\
\frac{\sin \mu z^{\prime} \sin \mu(H-z)}{\mu \sin \mu H}-\frac{\sinh \nu z^{\prime} \sinh \nu(H-z)}{\nu \sinh \nu H} & z>z^{\prime},\end{cases}
\end{gathered}
$$

$\overline{1}$ This quantity was denoted as $G_{2 \rightarrow 1}$ in Pázsit and Demazière (2010) 
where

$$
\begin{gathered}
\mu^{2}=\mu^{2}(\omega)=-\frac{1}{2}\left(\frac{\Sigma_{1}(\omega)}{D_{1}}+\frac{\Sigma_{2}(\omega)}{D_{2}}\right)+ \\
\frac{1}{2} \sqrt{\left(\frac{\Sigma_{1}(\omega)}{D_{1}}+\frac{\Sigma_{2}(\omega)}{D_{2}}\right)^{2}-\frac{4}{D_{1} D_{2}}\left(\Sigma_{1}(\omega) \Sigma_{2}(\omega)-\Sigma_{R} \nu \Sigma_{f 2}(\omega)\right)} \\
\nu^{2}=\nu^{2}(\omega)=\frac{1}{2}\left(\frac{\Sigma_{1}(\omega)}{D_{1}}+\frac{\Sigma_{2}(\omega)}{D_{2}}\right)+ \\
\frac{1}{2} \sqrt{\left(\frac{\Sigma_{1}(\omega)}{D_{1}}+\frac{\Sigma_{2}(\omega)}{D_{2}}\right)^{2}-\frac{4}{D_{1} D_{2}}\left(\Sigma_{1}(\omega) \Sigma_{2}(\omega)-\Sigma_{R} \nu \Sigma_{f 2}(\omega)\right)}
\end{gathered}
$$

we have:

$$
\begin{gathered}
G_{11}\left(z, z^{\prime}, \omega\right)=\left(D_{2} \nabla^{2}-\Sigma_{2}(\omega)\right) G_{i}\left(z, z^{\prime}, \omega\right), \\
G_{21}\left(z, z^{\prime}, \omega\right)=-\Sigma_{R} G_{i}\left(z, z^{\prime}, \omega\right), \\
G_{12}\left(z, z^{\prime}, \omega\right)=-\nu \Sigma_{f 2}(\omega)(1-\beta) G_{i}\left(z, z^{\prime}, \omega\right), \\
G_{22}\left(z, z^{\prime}, \omega\right)=\left(D_{1} \nabla^{2}-\Sigma_{1}(\omega)\right) G_{i}\left(z, z^{\prime}, \omega\right) .
\end{gathered}
$$

The equations for the adjoint Green's function are given by the adjoint twogroup frequency dependent diffusion operator. For the general case, the adjoint Green's function $\hat{\mathbf{G}}^{\dagger}\left(\overline{\mathbf{r}}, \overline{\mathbf{r}}^{\prime}, \omega\right)$ is also a $2 \times 2$ matrix, whose elements are determined by the equation:

$$
\hat{\mathbf{L}}^{\dagger}(\overline{\mathbf{r}}, \omega) \hat{\mathbf{G}}^{\dagger}\left(\overline{\mathbf{r}}, \overline{\mathbf{r}}^{\prime}, \omega\right)=\hat{\mathbf{I}} \cdot \delta\left(\overline{\mathbf{r}}-\overline{\mathbf{r}}^{\prime}\right)
$$

where $\hat{\mathbf{I}}$ is the $2 \times 2$ unit matrix and $\hat{\mathbf{G}}^{\dagger}\left(\overline{\mathbf{r}}, \overline{\mathbf{r}}^{\prime}, \omega\right)$ is given as:

$$
\hat{\mathbf{G}}^{\dagger}\left(\overline{\mathbf{r}}, \overline{\mathbf{r}}^{\prime}, \omega\right)=\left(\begin{array}{ll}
G_{11}^{\dagger} & G_{12}^{\dagger} \\
G_{21}^{\dagger} & G_{22}^{\dagger}
\end{array}\right)
$$

and

$$
\hat{\mathbf{L}}^{\dagger}=\hat{\mathbf{L}}^{\mathbf{T}}
$$

where the superscript $T$ denotes the transpose matrix operator. It can be shown (for a derivation, see the Appendix) that with the help of the adjoint Green's matrix, the noise can be expressed as:

$$
\delta \overline{\mathbf{\Phi}}^{\mathbf{T}}(\overline{\mathbf{r}}, \omega)=\int_{\mathbf{V}_{\mathbf{R}}} \overline{\mathbf{S}}^{\mathbf{T}}\left(\overline{\mathbf{r}}^{\prime}, \omega\right) \hat{\mathbf{G}}^{\dagger}\left(\overline{\mathbf{r}}^{\prime}, \overline{\mathbf{r}}, \omega\right) \mathbf{d} \overline{\mathbf{r}}^{\prime},
$$


where $\delta \overline{\mathbf{\Phi}}^{\mathbf{T}}(\overline{\mathbf{r}}, \omega)$ and $\overline{\mathbf{S}}^{\mathbf{T}}\left(\overline{\mathbf{r}}^{\prime}, \omega\right)$ denote row vectors.

Eq. (21) shows that in order to determine the fast and the thermal noise, one needs to know the first and the second column of the adjoint Green's matrix, respectively. These can be calculated from a single vector equation each, and this constitutes a certain advantage when only one of two group noises is to be determined. So far only the thermal noise was of interest, which can be calculated by the second column of the adjoint Green's matrix (Pázsit and Demazière, 2010). This vector is called, somewhat misleadingly, the "adjoint function" in the literature, with its two components being called the "fast adjoint" and the "thermal adjoin". In this terminology the fast adjoint means the transfer between the noise source in the fast group and the thermal noise, and the thermal adjoint the transfer between the thermal noise source and the thermal noise. In our general notations here, $G_{i j}^{\dagger}$ stands for the transfer from the noise source in group $i$ to the neutron noise in group $j$.

Since we will calculate both the fast and the thermal noise, neither the direct nor the adjoint Green's function technique will have any advantage over the other. Actually, as it is also easy to show, the direct and adjoint Green's functions obey the relationship

$$
G_{i j}^{\dagger}\left(z, z^{\prime}, \omega\right)=G_{j i}\left(z^{\prime}, z, \omega\right)
$$

Since in the simple model considered here (a homogeneous bare core), a solution can be obtained which is analytical in both arguments $z$ and $z^{\prime}$, the two functions are equivalent in all respect. In the illustrations which follow both will be used, the selection simply depending on which one brings out the phenomenon to be shown in a more effective way.

\section{Description of the cores used and specification of the perturba- tions}

As mentioned in the introduction, we will investigate the dynamic response of four different cores to various perturbations. These selected systems correspond to four different existing or planned reactor types, and they constitute a wide range of neutron spectra. Starting from the hardest spectrum to the softest, these systems are

- a MOX fuelled fast reactor

- a PWR

- a BWR

- a heavy water core (representing a CANDU).

The fact that the PWR has a harder spectrum than the BWR may appear 
somewhat surprising. The difference between these two cores is relatively small, and which of the two has harder or softer spectrum depends on the actual core loadings.

Material parameters, corresponding to these four different systems, and the spectral ratios $r=\frac{\phi_{1}^{0}}{\phi_{2}^{0}}$ are given in Table 1 . Here, the notation MOX stands for a fast system with MOX fuel, whose data were taken from Yoshioka et al (1998); the PWR and BWR data are generic data corresponding to Swedish LWRs; finally CANDU stands for a thorium+plutonium loaded heavy water reactor.

Table 1

\begin{tabular}{ccccc} 
Parameter & MOX & PWR & BWR & CANDU \\
\hline$H[\mathrm{~cm}]$ & 367.0 & 365.8 & 368.0 & 594.0 \\
$D_{1}[\mathrm{~cm}]$ & 1.4719 & 1.4375 & 1.7986 & 1.4068 \\
$D_{2}[\mathrm{~cm}]$ & 0.5364 & 0.3723 & 0.4342 & 0.8696 \\
$\nu \Sigma_{f 1}\left[\mathrm{~cm}^{-1}\right]$ & 0.0189 & 0.0056 & 0.0039 & 0.0007 \\
$\nu \Sigma_{f 2}\left[\mathrm{~cm}^{-1}\right]$ & 0.2839 & 0.1405 & 0.0740 & 0.0084 \\
$\Sigma_{a 1}\left[\mathrm{~cm}^{-1}\right]$ & 0.0168 & 0.0112 & 0.0074 & 0.0011 \\
$\Sigma_{a 2}\left[\mathrm{~cm}^{-1}\right]$ & 0.2818 & 0.1022 & 0.0581 & 0.0057 \\
$\Sigma_{R}\left[\mathrm{~cm}^{-1}\right]$ & 0.0083 & 0.0151 & 0.0131 & 0.0100 \\
$\lambda\left[\mathrm{s}^{-1}\right]$ & 0.0860 & 0.0881 & 0.0848 & 0.0848 \\
$\beta$ & 0.0040 & 0.0054 & 0.0058 & 0.0058 \\
$v_{1}[\mathrm{~cm} / \mathrm{s}]$ & $5.0806 \cdot 10^{7}$ & $1.8222 \cdot 10^{7}$ & $1.7549 \cdot 10^{7}$ & $4.3740 \cdot 10^{9}$ \\
$v_{2}[\mathrm{~cm} / \mathrm{s}]$ & $2.8216 \cdot 10^{5}$ & $4.1388 \cdot 10^{5}$ & $3.9040 \cdot 10^{5}$ & $2.4190 \cdot 10^{6}$ \\
$r=\frac{\phi_{1}^{0}}{\phi_{2}^{0}}$ & 33.83 & 6.77 & 4.43 & 0.56
\end{tabular}

Steady-state parameters for each reactor type.

The modelling of the perturbation, as seen in Eqs. (5) and (6), requires the knowledge of fluctuations of the various macroscopic two-group cross sections. We assume that the propagating perturbation in all four reactors, including the BWR, consists of the inlet temperature fluctuations of the coolant, and the corresponding variations of all the cross sections were calculated by using system codes. This constitutes a novelty as compared to most of the previous work in the field. In a one-group approach, in most works, it was assumed that a particular perturbation manifests itself in the change of one single cross section. The two most important applications have been the vibration of control rods and the effect of inlet temperature fluctuations of the coolant, both referring to PWRs. In both cases it was assumed that only the absorption cross section is perturbed, and immediate assumptions could be made on the 
analytical form of the space and frequency dependence of $\delta \Sigma_{a}(x, \omega)$. Only the functional form of the expression, but not its magnitude was of interest, in order to extract the necessary information (such as to locate the position of a vibrating absorber). In the case of boiling water reactors, only the perturbation of the removal cross section was considered, hence again it was sufficient to define $\delta \Sigma_{R}(x, \omega)$ as a propagating perturbation, again without any need of specifying its magnitude.

In the present case, the change in all cross sections was calculated for a change of $1{ }^{\circ} \mathrm{C}$. The choice of this temperature change is of course arbitrary, hence the overall magnitude of the cross section fluctuations still can be left arbitrary. However, the relative amplitudes of the various cross section fluctuations in relation to each other are correctly determined, which hence provides a realistic modelling of such a perturbation. As it will be seen, the internal relationships of the various cross section perturbations have an influence on the induced noise.

These internal relationships were determined by using the in-core fuel management neutronic system code SIMULATE-3 Studsvik Scandpower (2001) to determine the change of the various two-group cross sections to a unit change in the coolant temperature. This is given in Table 2 .

Table 2

\begin{tabular}{ccccc} 
Parameter & MOX & PWR & BWR & CANDU \\
\hline$\delta \nu \Sigma_{f 1}\left[\mathrm{~cm}^{-1}\right]$ & $-2.8 \cdot 10^{-6}$ & $-2.8 \cdot 10^{-6}$ & $-5.0 \cdot 10^{-6}$ & $-2.8 \cdot 10^{-6}$ \\
$\delta \nu \Sigma_{f 2}\left[\mathrm{~cm}^{-1}\right]$ & $-5.0 \cdot 10^{-5}$ & $-5.0 \cdot 10^{-5}$ & $-2.8 \cdot 10^{-5}$ & $-5.0 \cdot 10^{-5}$ \\
$\delta \Sigma_{R}\left[\mathrm{~cm}^{-1}\right]$ & $-5.2 \cdot 10^{-5}$ & $-5.2 \cdot 10^{-5}$ & $-1.2 \cdot 10^{-4}$ & $-5.2 \cdot 10^{-5}$ \\
$\delta \Sigma_{a 1}\left[\mathrm{~cm}^{-1}\right]$ & $-5.0 \cdot 10^{-6}$ & $-5.0 \cdot 10^{-6}$ & $-1.5 \cdot 10^{-5}$ & $-5.0 \cdot 10^{-6}$ \\
$\delta \Sigma_{a 2}\left[\mathrm{~cm}^{-1}\right]$ & $-6.0 \cdot 10^{-5}$ & $-6.0 \cdot 10^{-5}$ & $-5.8 \cdot 10^{-5}$ & $-6.0 \cdot 10^{-5}$
\end{tabular}

Cross section perturbations induced by a small $\left(1^{\circ} \mathrm{C}\right)$ perturbation in the coolant inlet temperature for the four different reactor types.

\section{Static fluxes and adjoints}

The static fast and thermal fluxes are shown in Fig. 1 for all four systems. The static adjoints are shown in Fig. 2. The static fluxes show clear spectral differences, and they demonstrate that the sequence of fast to thermal systems is as described in the previous section, with the MOX loaded fast system having the hardest and the heavy water moderated CANDU reactor the softest. As expected, the static adjoints, showing the importance of the fast and thermal source neutrons, show a reversed sequence of the spectral characteristics. 

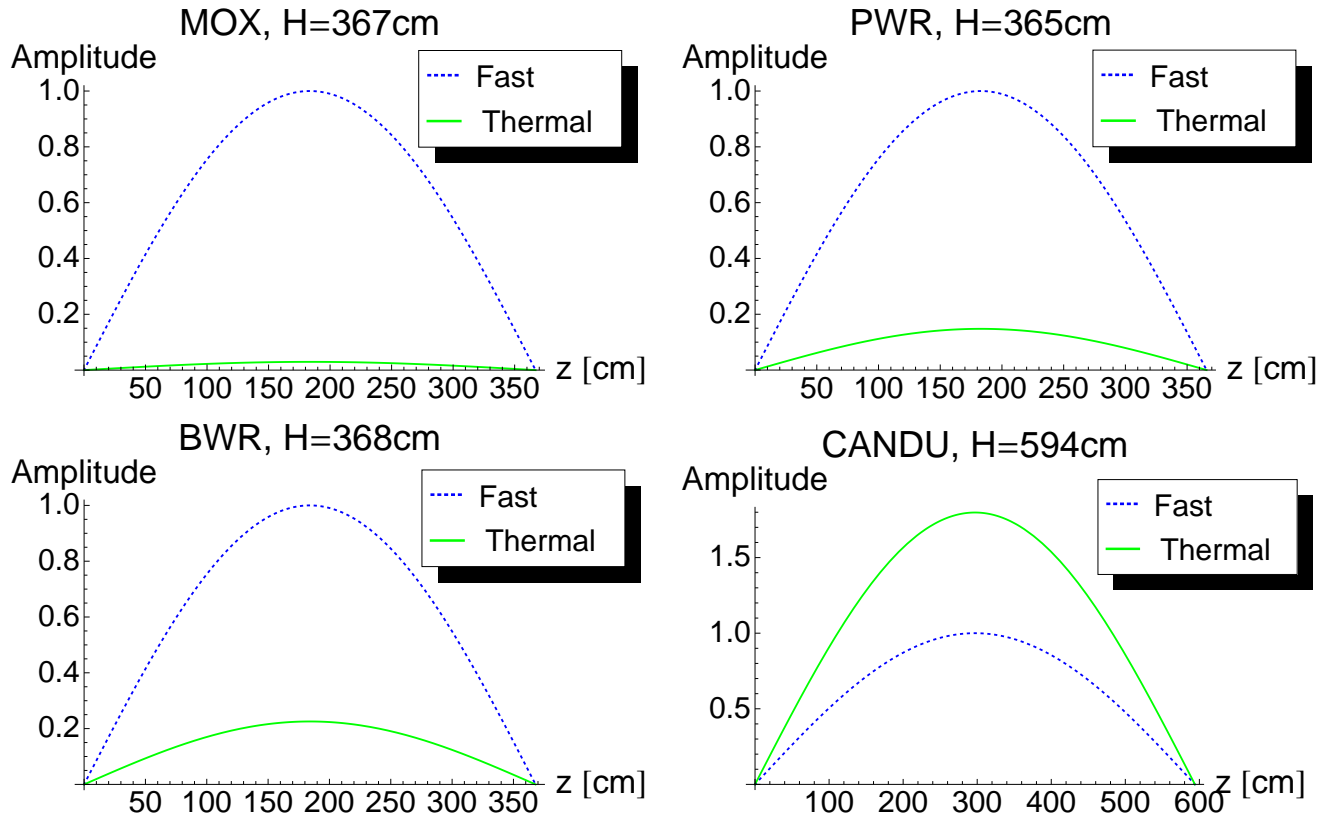

Fig. 1. Static fluxes for: a fast reactor with MOX fuel (upper left figure); PWR (upper right figure); BWR (lower left figure); and CANDU reactor (lower right figure).
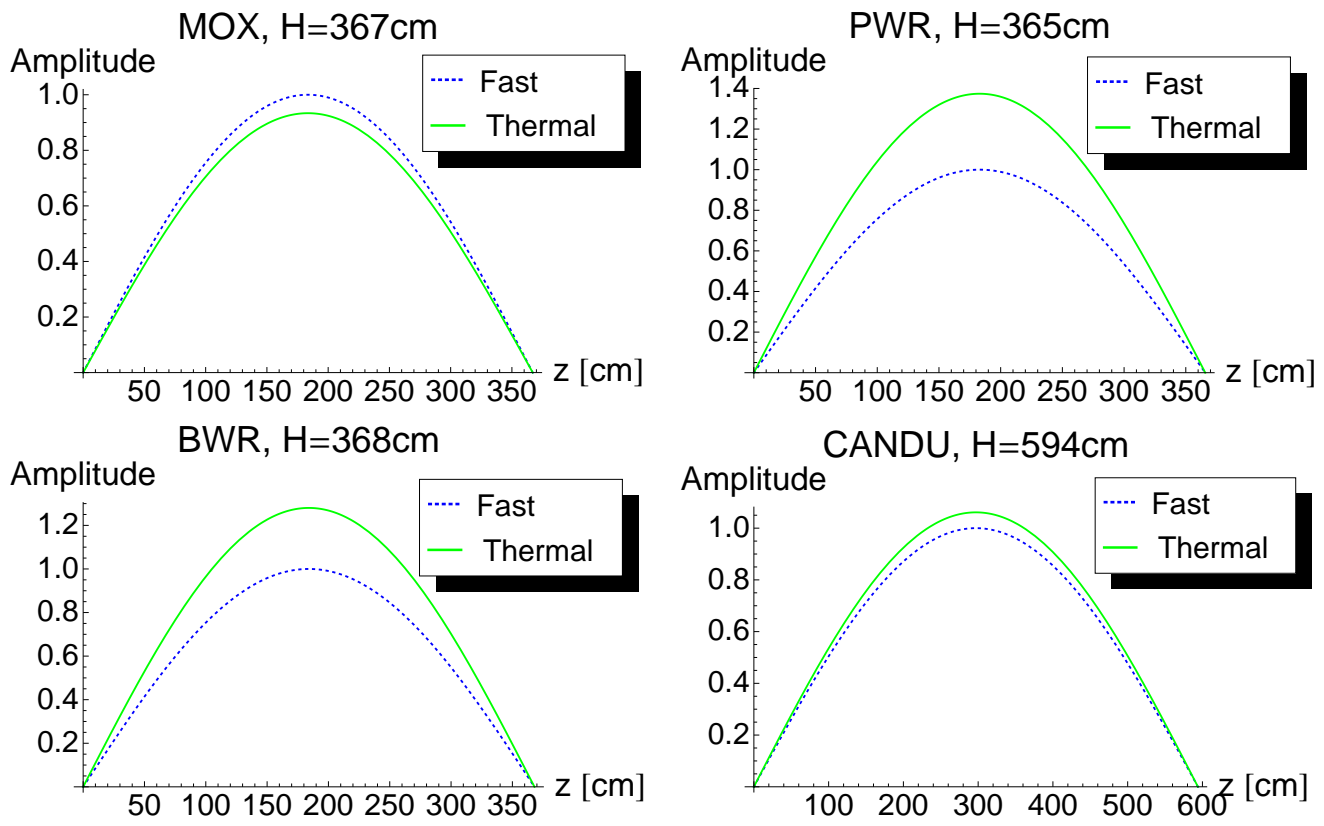

Fig. 2. Static adjoints for: a fast reactor with MOX fuel (upper left figure); PWR (upper right figure); BWR (lower left figure); and CANDU reactor (lower right figure). 


\section{$5 \quad$ Frequency dependence of the direct Green's functions}

The frequency dependence of the four components of the Green's matrix is shown in Fig. 3. for $z=z^{\prime}=H / 2$, i.e. a central perturbation and central detector. These plots show a close resemblance to those in Jonsson and Pázsit (2011) which referred to MSR cores with similar spectral properties. The frequency dependence of the Green's functions follows that of the zero power reactor transfer function. As expected, the upper break frequency is lower for the heavy water moderated CANDU reactor than for the other cores. It is also seen that the four components are separated into two groups. The components $G_{11}$ and $G_{12}$, which represent the noise in the fast group induced by fast and thermal noise sources, respectively, have a similar amplitude, and they are separated from the components $G_{21}$ and $G_{22}$, which represent the thermal noise, and which also have similar amplitudes. The relationship between the amplitudes of these two groups follows that of the spectral ratio between the static fluxes. The separation between the two fast noise components and the two thermal noise components is largest for the MOX core, then it gradually decreases for the PWR and the BWR cores. For the CANDU core, the thermal noise is larger than the fast noise.
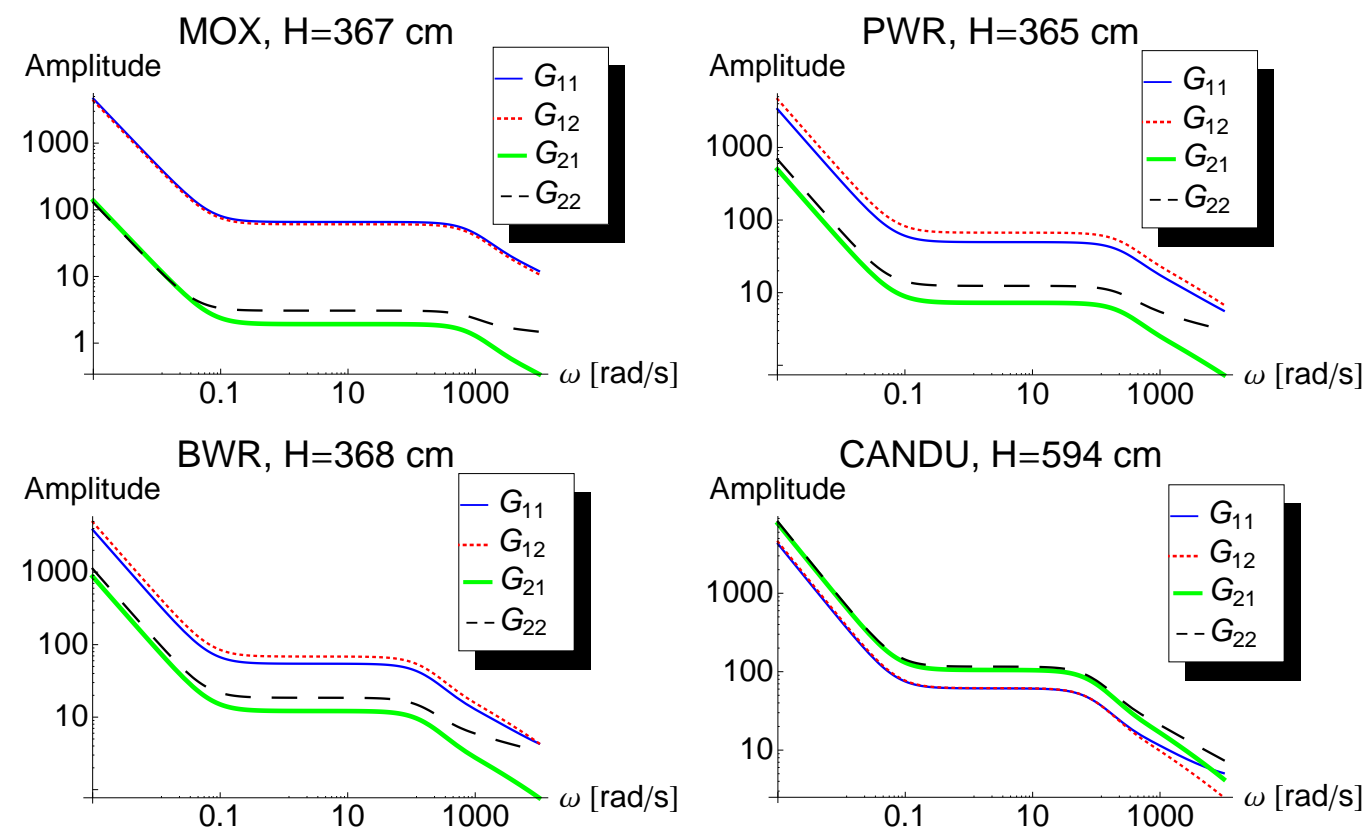

Fig. 3. Frequency dependence of the amplitude of the direct Green's function components: MOX (upper left figure), PWR (upper right figure), BWR (lower left figure) and CANDU reactor (lower right figure) $\left(z=z^{\prime}=H / 2\right)$.

These results show that even for the light water cores, but in particular for the fast spectrum core, the neutron noise induced by a unit strength perturbation 
(either in the fast or in the thermal group) is significantly larger in the fast group than in the thermal group. For the MOX core the fast noise is more than an order of magnitude larger than in the thermal group. This indicates that even if detection of the fast neutrons is far less effective than that of the thermal neutrons, in fast reactors the use of the fast neutron noise could be a very useful complement to that of the traditional thermal neutron noise.

It is also interesting to note that in the fast and light water reactors, the frequency dependence of the component $G_{22}$ deviates from that of the other three components at high frequencies (above the upper break frequency). It does not decrease as $1 / \omega$ as the other three components do, its decay is much slower. The reason for this is the presence of the local component. Since the plots in Fig. 3 show the Green's functions components at $z=z^{\prime}$, the detection point is at the maximum of the local component (cf. Fig. 5 in the next Subsection). As it is known from the literature, and which is also easy to see from Eqs (12) and (13), the break frequency of the local root $\nu^{2}(\omega)$ is very much higher than that of the global root $\mu^{2}(\omega)$, and is practically constant at the global break frequency $\beta / \Lambda$. Hence, when the amplitude of the global component decreases below the local component, this latter will determine the amplitude which remains nearly constant. This is the same phenomenon as the behaviour of the phase of the neutronic response in a reactor oscillator experiment in the Norwegian heavy water reactor NORA, as described in Bell and Glasstone (1970), pp 488-489.

Curiously, for the case of the CANDU core, in addition to the component $G_{22}$ whose high frequency behaviour deviates from the others, even that of the component $G_{11}$ deviates to the same direction, even more visibly than $G_{22}$. This is in correspondence with the fact that for the CANDU case, there appears to be a small local component both in $G_{11}$ and $G_{22}$. This is seen in Figs 4 and 5. These show the adjoint Green's functions, but as was mentioned the diagonal components are equivalent with the direct Green's functions.

\section{Space dependence of the adjoint Greens functions}

Since in most work in the past the nature of the local component, and hence the space dependence of the transfer functions was performed by using the dynamic adjoint, in this subsection we will also use the component of the adjoint matrix. As mentioned earlier, in the past only the thermal neutron noise was of interest, for whose calculation it was sufficient to use the second column of the adjoint matrix. Here we will display the first and the second column of the adjoint matrix separately. The two components of the first column, $G_{11}^{\dagger}$ and $G_{21}^{\dagger}$ give the fast neutron noise induced by a localised perturbation in the fast and the thermal group, respectively, whereas the two components of 
the second column, $G_{12}^{\dagger}$ and $G_{22}^{\dagger}$ give the same for the thermal noise. It is these two components which correspond to the components $\Psi_{1}^{\dagger}$ and $\Psi_{2}^{\dagger}$ of the so-called thermal adjoint.

Again, in correspondence with the literature, the quantitative work will be restricted to the plateau frequency region $\lambda<\omega<\beta / \Lambda$. The frequency value $\omega=2 \mathrm{rad} / \mathrm{sec}$ will be used throughout.
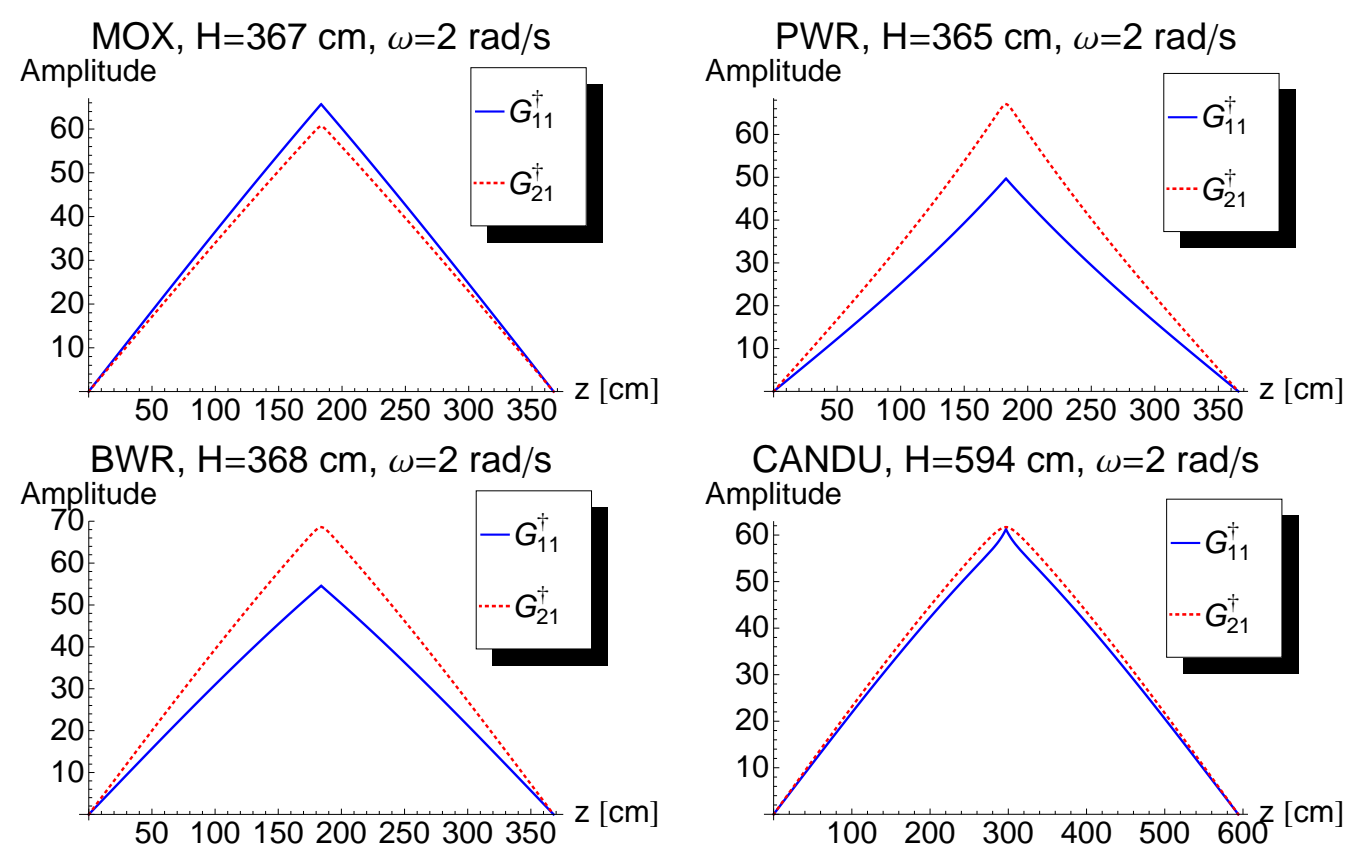

Fig. 4. Space dependence of the amplitude of the components of the fast adjoint: MOX (upper left figure), PWR (upper right figure), BWR (lower left figure) and CANDU reactor (lower right figure) for $\omega=2 \mathrm{rad} / \mathrm{s}$ and $z^{\prime}=H / 2$.

The space dependence of the amplitude of the components of the fast adjoint function is shown in Fig. 4. Since the figure shows the case of power reactors at the plateau region, there is a seeming deviation from the point kinetic behaviour. In these Green's function components there is no visible occurrence of the local component, except, as was mentioned, in $G_{11}^{\dagger}$ of the CANDU core. It has to be added that the range of the local component (equal to the thermal diffusion length) is much larger in a heavy water system than in a light water reactor. Hence the local component is much less "local" in a CANDU reactor than in an LWR, which also makes it more difficult to discern it from the plot of the adjoint Green's function.

The space dependence of the amplitude of the thermal adjoints for the four cores is shown in Fig. 5. These show a resemblance to earlier calculations in the literature with a strong local component clearly displayed for the fast and the light water cores. The local component is the largest for the fast spectrum core. 
Somewhat surprisingly it is hardly visible for the CANDU core. One reason could be the earlier mentioned large range of the local component in heavy water systems, which makes the presence of the local component more difficult to see on the plot; but even so, the local component appears to have a smaller weight in HWRs than in LWRs. There appear to be no reported calculations in the literature of the transfer or adjoint function of CANDU cores, hence it is not possible to say whether this observation agrees or disagrees with other work in the field.
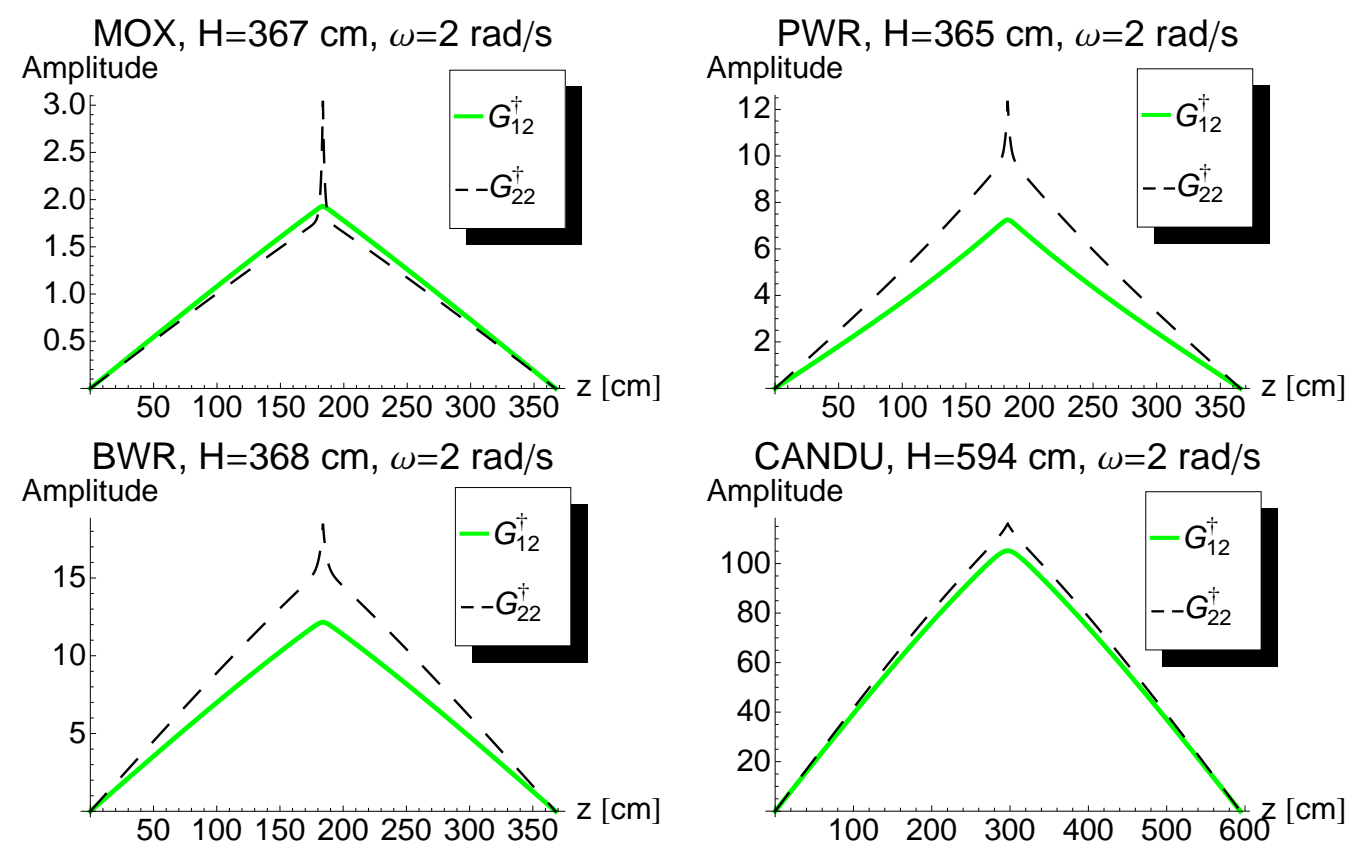

Fig. 5. Space dependence of the amplitude of the components of the thermal adjoint: MOX (upper left figure), PWR (upper right figure), BWR (lower left figure) and CANDU reactor (lower right figure) for $\omega=2 \mathrm{rad} / \mathrm{s}$ and $z^{\prime}=H / 2$.

\section{Investigation of the propagation noise}

The main motivation for the present study was to investigate the space and frequency dependence of the propagation noise, i.e. the neutron noise induced by a propagating perturbation, in systems of various spectral properties, and whenever feasible, compare it with the result of similar calculations performed recently by Jonsson and Pázsit (2011). The origin of the interest in propagating noise lies in the characteristics of the neutron noise induced by inlet temperature fluctuations propagating with the coolant of a PWR, and the noise induced by the two-phase flow, propagating with the coolant of a BWR. The recent interest arose in connection with molten salt reactors where density and material inhomogeneities may propagate with the molten salt and hence can give rise to neutron noise. 
As usual, the temperature, void etc. perturbations are represented by the fluctuations of some group constants. The propagating property is expressed as

$$
\delta \Sigma(z, t)=\delta \Sigma(z=0, t-z / v)
$$

where $v$ is the velocity of the propagation. In the frequency domain, (22) will read as

$$
\delta \Sigma(z, \omega)=\delta \Sigma(z=0, \omega) e^{-i \omega \frac{z}{v}}
$$

This form is then substituted into the expressions for the noise source, Eqs (5) and (6), which then in turn can be integrated either with the direct Green's function as in (10) or with the adjoint Green's function, Eq. (21), to obtain the group noises. These two methods are completely equivalent and require identical effort. In this work the adjoint function was used to calculate the noise, without any specific reason.

\subsection{Space dependence of the propagation noise}

The noise calculations will be performed and discussed in two steps. First, it will be assumed that only the thermal absorption cross section is perturbed. This is because we want to relate the results to earlier ones in the literature, and these were so far always calculated by considering the perturbation of only one cross section, either that of the thermal absorption cross section or the removal cross section. This is necessary for such a comparison since, as it will be seen shortly, when taking into account the case of several cross sections being perturbed, the structure of the propagation noise is significantly changed.

The space dependence of the fast and thermal propagation noise induced by fluctuations of the thermal absorbing cross section is shown in Fig. 6 for the four core types investigated. These plots have a form similar to those of the one-group calculations of Pázsit and Dykin (2010) in a PWR and the twogroup calculations of Pázsit and Jonsson (2010) in molten salt systems. The reason for the spatial oscillations of the noise is the same as described in the previous publications, i.e. an interference phenomenon between the point kinetic and space dependent components. The spatial structure of the fast and the thermal noise is the same, which is also in accordance with the previous results. It is seen that the spectral ratio of the noise is similar to that of the static fluxes and can be also directly related to the spectral ratio of the corresponding elements of the direct Green's function (Fig. 3). For the MOX core the thermal noise is vanishingly small as compared to the fast noise, and the thermal noise was multiplied in the figure with a factor 6 for better visibility.

The strong spatial tilt of the space dependence of the noise, observed in several 
cases of MSR calculations, published in (Pázsit and Jonsson, 2010) cannot be seen here. This effect seems therefore to be related to the propagating fuel, or the material properties of other cores such as thorium loaded ones.
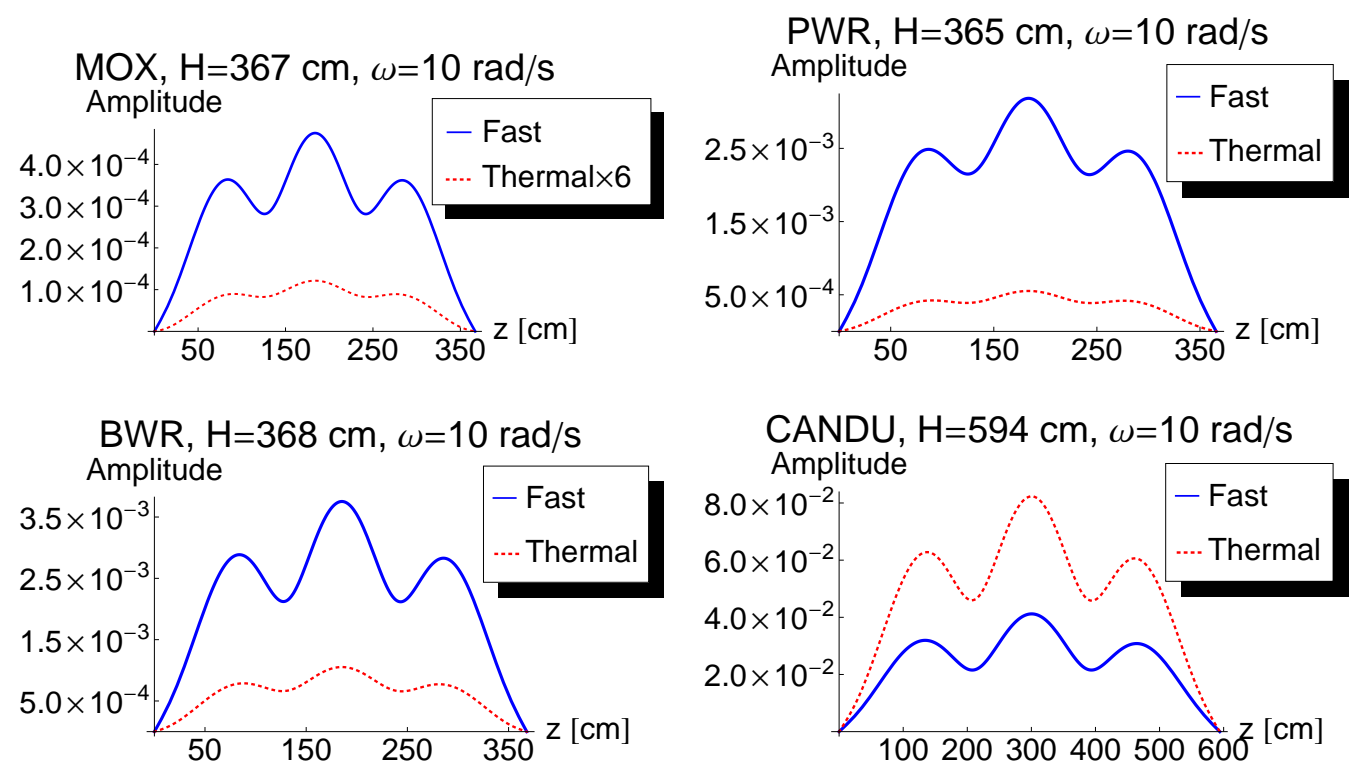

Fig. 6. Space dependence of the neutron noise induced by propagating perturbation of the thermal absorption cross-section only: in a MOX (upper left figure), in a PWR (upper right figure), in a BWR (lower left figure) and in a CANDU reactor (lower right figure) for $\omega=10 \mathrm{rad} / \mathrm{s}$ and $v=H / 2 \mathrm{~cm} / \mathrm{s}$. For the MOX core, the thermal noise was multiplied by a factor 6 for better visibility.

The space dependence of the propagation noise as induced by perturbations of all cross sections is shown in Fig. 7. The fluctuations of the individual cross sections were calculated by the ICFM system code SIMULATE-3 by considering the perturbation of the inlet coolant temperature in both the PWR and the BWR core. Since, unlike for the PWR and BWR case, we have access neither to system codes nor to input decks, no similar calculations were performed for the fast MOX system and the thermal CANDU core. Instead, the same internal ratio of the various cross section perturbations was used as for the PWR. In view of the fact that a significant difference was found between the cases when the fluctuation of only one cross section generates the neutron noise and when the fluctuation of several cross sections is taken into account, and in view of the fact that the latter corresponds to the real case much better, it would be advisable to perform similar calculations for fast and heavy water systems as well. Fast systems can be treated by the system code ERANOS, whose application to this problem is planned in later work. 

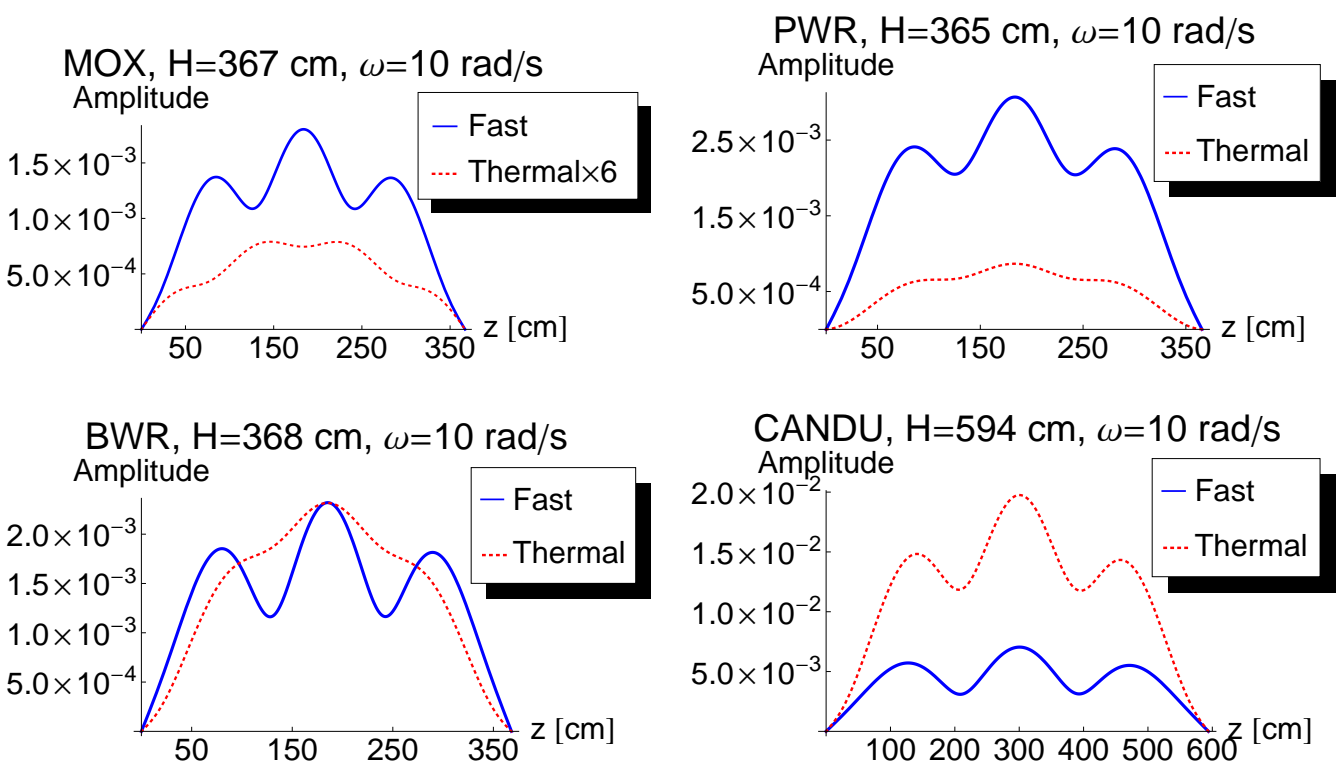

Fig. 7. Space dependence of the propagation noise induced by the fluctuations of all cross-sections in the four cores considered, for $\omega=10 \mathrm{rad} / \mathrm{s}$ and $v=H / 2 \mathrm{~cm} / \mathrm{s}$. For the MOX core, the thermal noise was multiplied by a factor 6 for better visibility.

The characteristics of the propagation noise show a number of interesting features. The fast noise looks similar to that of the previous case, but the thermal noise displays several differences. One of these is the case of the MOX core, where the oscillating structure of the thermal noise is changed and became different from that of the fast noise. The maxima and the minima changed place, and the spatial oscillations of the fast and the thermal noise are out of phase.

The explanation is that when fluctuations of several cross sections take place simultaneously, their effect adds up with different signs and different weights in the resulting noise. The sign differences can be seen in Eqs (5) and (6). These are simply due to the fact that different processes contribute differently to the neutron balance, through representing generation or destruction of the neutrons in the corresponding groups. The different weighting is related to the fact that the fast and thermal noise sources are contributing to the propagation noise through a weighting by the different components of the Green's function (Eq. 10) (or, equivalently, by the components of the adjoint Green's function). As was seen earlier, the amplitude of different components shows also a significant variation. Moreover, the phase of the different Green's function components is also different. All these factors contribute to the fact that the structure of the propagation noise (and also that of the neutron noise induced by other perturbations) is more complicated for the case when the fluctuation of all cross sections is taken into account. This will be more visible and discussed further in the next subsection. 
Another difference between Figs 6 and 7 is that in the latter, the thermal noise is much larger in the BWR than in the case of pure thermal absorption cross section perturbations. The explanation is that unlike in the other cores, in the BWR, an increase of the coolant temperature leads to an increase of the void fraction, with the result that the change in the removal cross section becomes much larger than that of the thermal absorption cross section. On its turn, perturbation of the removal cross section affects the thermal noise much more than the fast noise, although the pure contribution from the removal cross section is comparable for the fast and the thermal noise. This is because in the thermal noise, the component due to the removal cross section dominates over the noise induced by the other cross section fluctuations. In the fast noise, the noise induced by the removal cross section and the noise by the other cross section fluctuations are of comparable magnitude but opposite phase, hence there is no increase of the noise by adding the fluctuations induced by the removal cross section.

\subsection{Frequency dependence of the propagation noise}

The same sequence will be followed here as for the space dependence. First the frequency dependence of the amplitude of the propagation noise, induced solely by the fluctuations of the thermal absorption cross section, will be calculated. The result is shown in Fig. 8 for the four cores. Several features of these spectra show similarities with the corresponding MSR calculations by Jonsson and Pázsit (2011). One is the spectral ratio of the noise, which also follows from the results shown earlier in this paper for the Green's functions (Fig. 3) and the space dependence of the propagation noise (Fig. 6). Two further such features are the somewhat deeper sinks in the fast noise than in the thermal noise, and the slower decay of the spectra with increasing frequency in the thermal noise than in the fast noise.

Both of these latter features depend on the fact that the thermal noise is affected by the presence of the local component, whereas the weight of the local component is vanishingly small in the transfer function components which are used to calculate the fast noise. Regarding the depth of the sinks, the local component, similarly to the pure space dependent component, does not have a sink structure, hence its presence makes the sinks relatively shallower. Concerning the decay of the spectra with increasing frequency, the part of the noise which is due to the local component, will decay slower than the global component (consisting of the sum of the reactivity and the space dependent part), for two reasons. One of these relates to the frequency dependence of the amplitude of the corresponding component of the transfer function. The amplitude of the local component decays much slower in frequency than that of the global component. This is the same phenomenon as the one behind the 

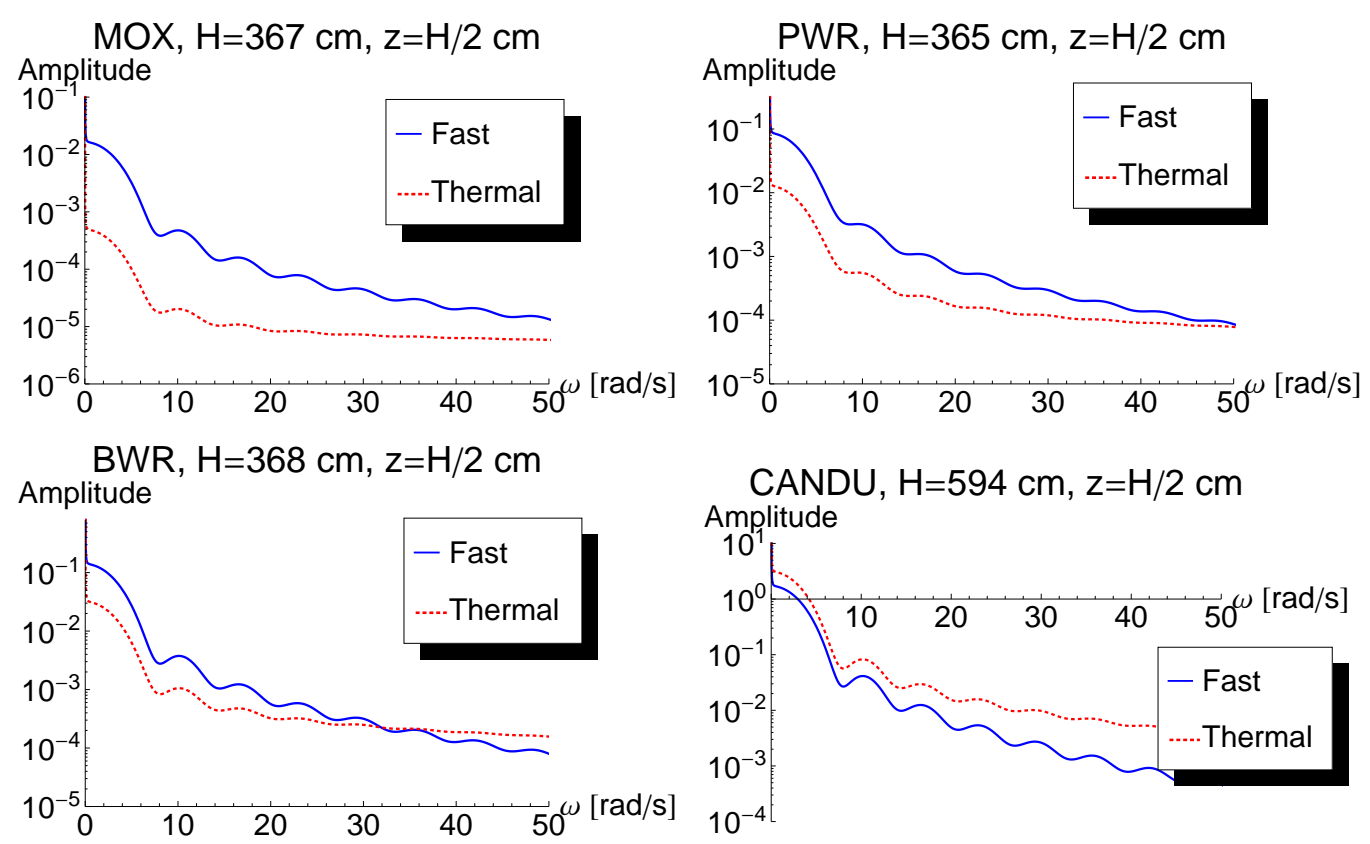

Fig. 8. Frequency dependence of the neutron noise induced by propagating temperature perturbation in absorption cross-section only in MOX reactor (upper left figure), in PWR (upper right figure), in BWR (lower left figure) and in CANDU reactor (lower right figure) for $z=H / 2 \mathrm{~cm}$ and $v=H / 2 \mathrm{~cm} / \mathrm{s}$.

high frequency tail of $G_{22}$, described earlier. The other reason is related to the integral of the transfer functions with the noise source. As is seen from Eq. (23), the propagating perturbation represents a function which oscillates in space, and the oscillation frequency increases with increasing frequency. The integral of any smooth function with a more and more rapidly oscillating function decreases monotonically. This decrease is much faster for the global component, which varies smoothly in space with a relaxation length comparable with the core size, than for the local component, whose relaxation length is much shorter. These two effects together explain the slower decay of the thermal noise with increasing frequency as compared to the fast noise.

The next step is the calculation of the propagation noise, induced by the perturbation of the coolant temperature, affecting all cross sections, as described in the previous Subsection. The results are shown in Fig. 9. The structure of these spectra is significantly more complicated and irregular than in the previous case when the noise was induced only by the fluctuations of the thermal absorption cross section. Some general properties (such as the spectral ratio of the noise components, the less pronounced sink structure of the thermal noise, its slower decay in frequency as compared to the fast noise, etc. ) are though preserved, although in a less clear form. These general features have the same reason as for the case of the noise generated only by one cross section fluctuations, shown in Fig. 8. However, the sink structure is much diminished and irregular even for the fast noise, and there are some peculiar deep dips in 

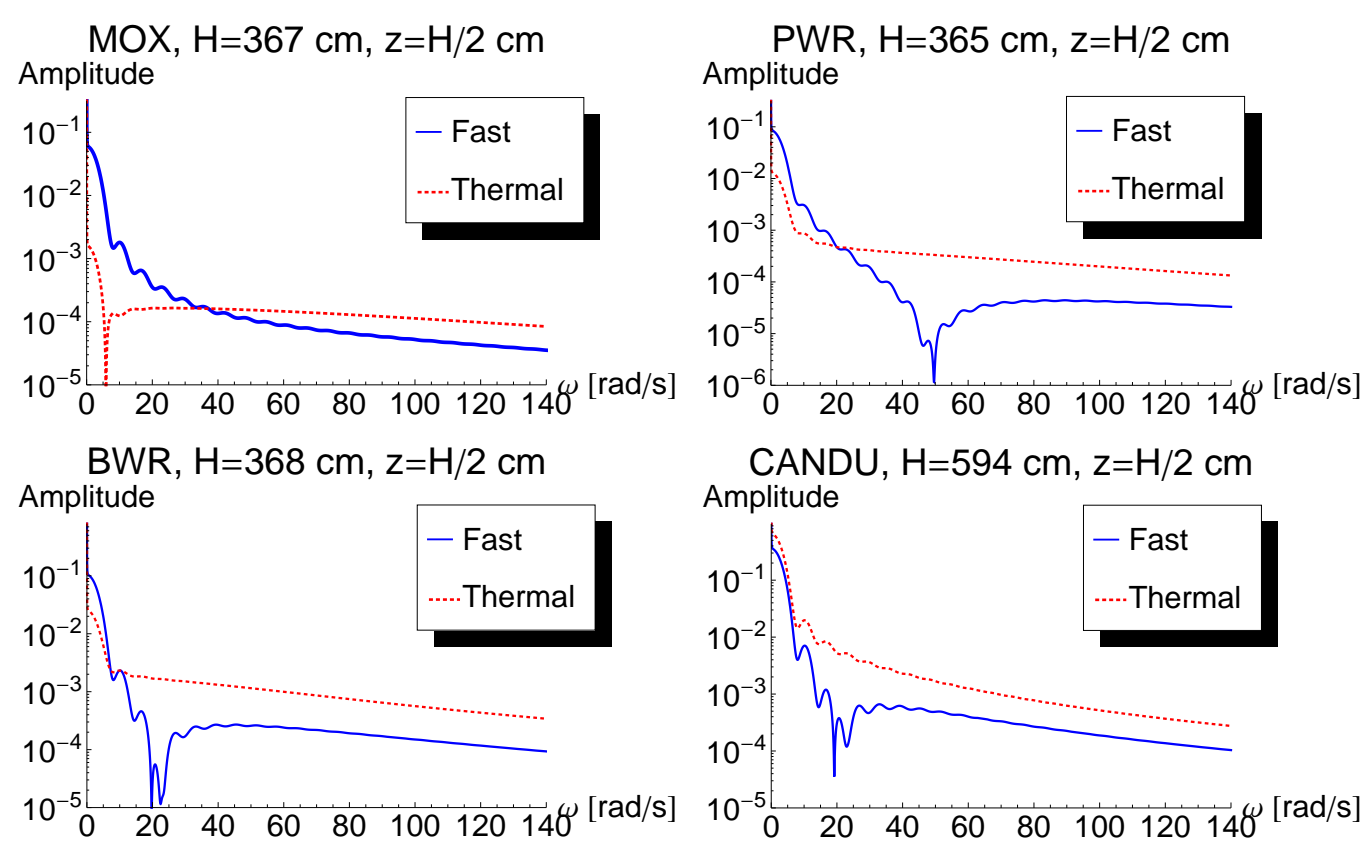

Fig. 9. Frequency dependence of the neutron noise induced by propagating temperature perturbation in MOX (upper left figure), in PWR (upper right figure), in BWR (lower left figure) and in CANDU reactor (lower right figure) for $z=H / 2 \mathrm{~cm}$ and $v=H / 2 \mathrm{~cm} / \mathrm{s}$.

either the fast or the thermal noise at a given frequency in all systems. The frequency of this dip and its character is though different for the four different systems.

The seemingly irregular sink structure and the single deep sinks in the spectra can all be understood by considering the contributions of the various cross section fluctuations separately, and in some cases splitting the contribution further to components due to the local and the global components of the noise. Namely, the structure of the noise is the result of the interplay of these various components, and although each of them has either a smooth or regular periodic structure, their interplay leads to the irregularly looking behaviour.

As an example, we shall take a closer look at the structure of the frequency dependence of the propagation noise for the case of a PWR shown in Fig. 9 (top right figure) where the amplitude of the fast noise has a deep sink around $50 \mathrm{rad} / \mathrm{s}$. All cross sections are affected by the temperature fluctuations of the coolant: the absorption cross sections in the fast and thermal energy groups, the fission cross sections in the fast and thermal groups, and the removal cross section. We first split the total neutron noise into five components, each induced by the perturbation of one of the cross sections. The amplitude and the phase of the five components is shown in Figs. 10-11. For better visibility of the entire noise structure, the amplitudes are given separately in Fig. 10, whereas Fig. 11 represents only the most interesting parts of the curves. 
PWR, $\mathrm{H}=365 \mathrm{~cm}, \mathrm{z}=\mathrm{H} / 2 \mathrm{~cm}, \mathrm{FG}$ Amplitude

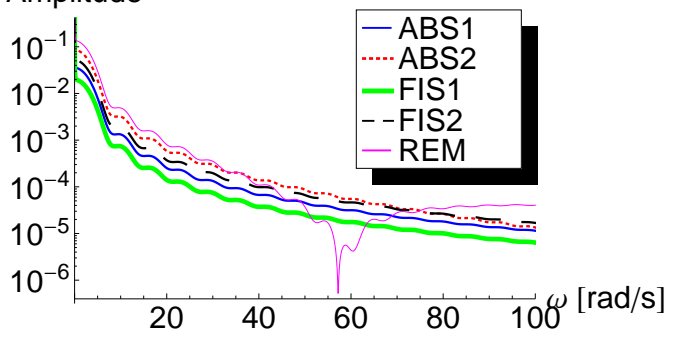

PWR, $\mathrm{H}=365 \mathrm{~cm}, \mathrm{z}=\mathrm{H} / 2 \mathrm{~cm}, \mathrm{TG}$

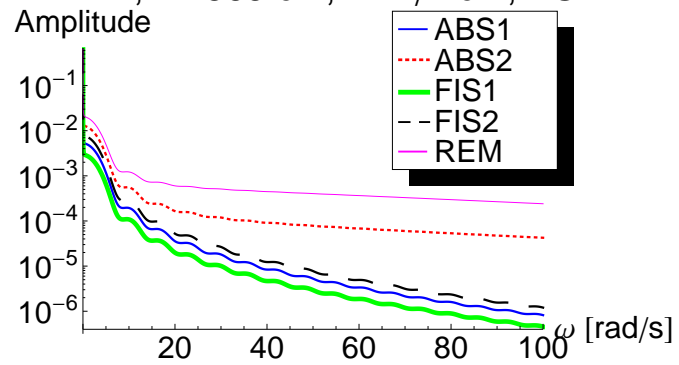

Fig. 10. Frequency dependence of the amplitude of the neutron noise induced by propagating temperature perturbation in ABS1, ABS2 FIS1, FIS2 and REM separately for a PWR in fast (left figure) and thermal (right figure) groups, respectively for $z=H / 2 \mathrm{~cm}$.

PWR, $\mathrm{H}=365 \mathrm{~cm}, \mathrm{z}=\mathrm{H} / 2 \mathrm{~cm}, \mathrm{FG}$ Amplitude

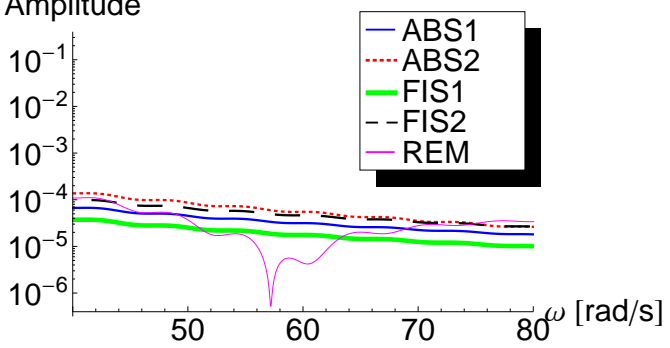

PWR, $\mathrm{H}=365 \mathrm{~cm}, \mathrm{z}=\mathrm{H} / 2 \mathrm{~cm}, \mathrm{FG}$ Phase

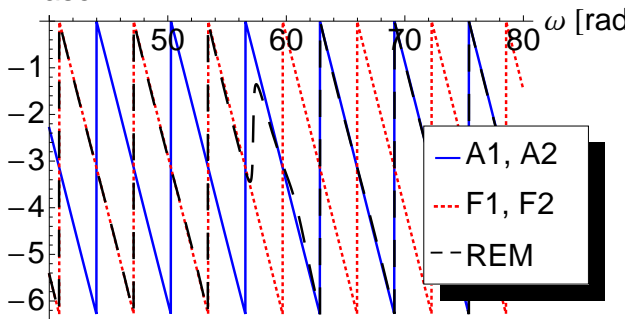

PWR, $\mathrm{H}=365 \mathrm{~cm}, \mathrm{z}=\mathrm{H} / 2 \mathrm{~cm}, \mathrm{TG}$

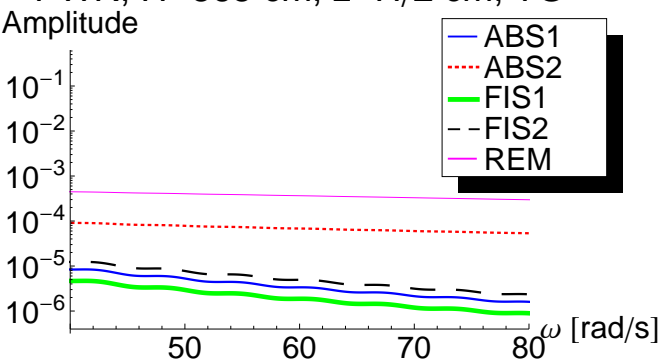

PWR, $\mathrm{H}=365 \mathrm{~cm}, \mathrm{z}=\mathrm{H} / 2 \mathrm{~cm}, \mathrm{TG}$ Phase

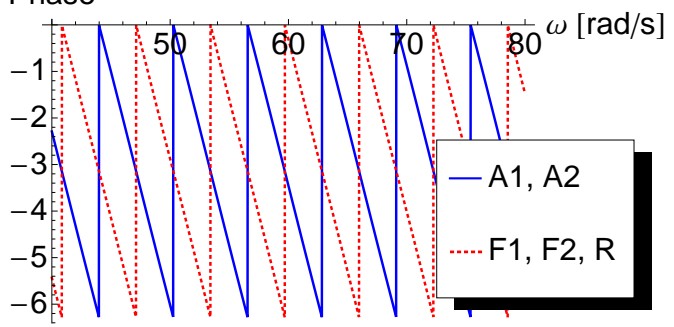

Fig. 11. Frequency dependence of the amplitude and phase of the neutron noise induced by propagating temperature perturbation in fast absorption cross section (ABS1-component), thermal absorption cross section (ABS2-component), fast fission cross-section (FIS1-component), thermal fission cross-section (FIS2-component) and removal cross-section (combined REM-component) separately for a PWR in fast (left figures) and thermal (right figures) groups (denoted as FG and TG), respectively for $z=H / 2 \mathrm{~cm}$.

The phase of the noise does not have much information except the phase relationship between the different components. Some components are in-phase, some out-of-phase. The monotonically decreasing slope just follows that of the perturbation, as is discussed in (Pázsit and Dykin, 2010). The in- or out-ofphase property explains if there is a constructive or destructive interference between the different components of the noise. 
There is more information in the amplitude of the noise components. As Fig. 10 shows, each component, except the one induced by the removal cross section fluctuations, shows a regular sink structure, similar to that observed in earlier work. However, since some components are out-of-phase with each other (for example, the components ABS1, ABS2, REM11 and REM21 with respect to the components FIS1, FIS2, REM12 and REM22), the sum of the components has a less pronounced sink structure. In addition, the position of the sink frequencies can also slightly vary for the different components, due to the different interplay of the reactivity and the space dependent components, which is due to differences in the corresponding Green's function components. It is also seen that, in particular in the thermal noise, some components decay slower than some others.

It is also seen that the similar deep sink, which is observed in the total fast noise in Fig. 9, is present in the noise component due to the removal cross section fluctuations. In order to understand the reason for this, we need to split even this component into further components. Since the fluctuations of the removal cross section appear in both the fast and the thermal noise source, two different components of the direct or adjoint Green's function are involved in the calculation of the neutron noise, taken with opposite sign. The large dip in the fast noise around $50 \mathrm{rad} / \mathrm{s}$ induced by the removal cross section is due to the interplay between these two Green's function components, but are even related to the local and the global components of the noise. This is illustrated first by splitting up the noise partly for the different Green's function components, i.e. $G_{11}$ and $G_{12}$, and partly into local and global components. The splitting up of the noise for the two different Green's function components for the removal cross section is shown in Figs. 12-13. For the same reason as before, the amplitudes are given separately in Fig. 12 .
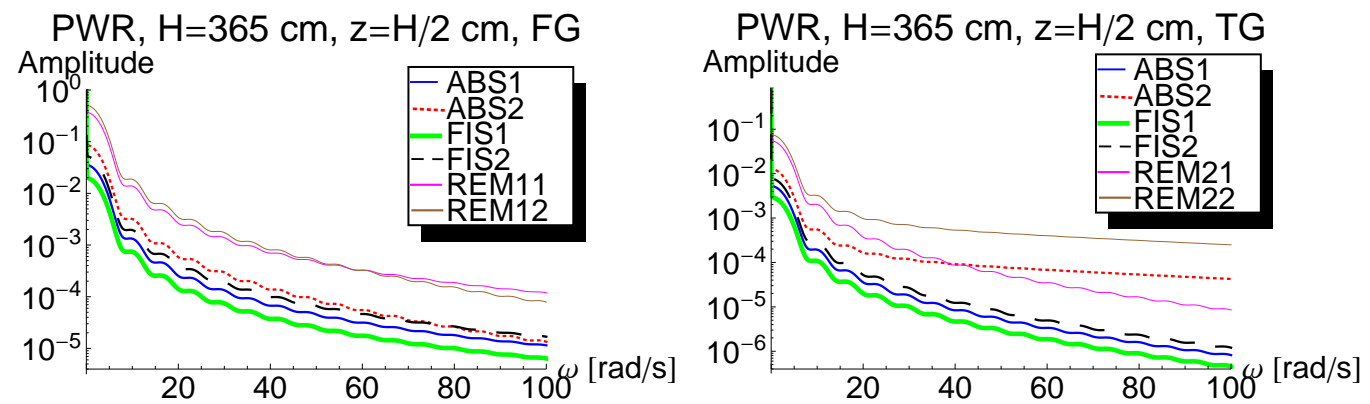

Fig. 12. Frequency dependence of the amplitude of the neutron noise induced by propagating temperature perturbation in ABS1, ABS2 FIS1, FIS2 and REM separately for a PWR in fast (left figure) and thermal (right figure) groups, respectively for $z=H / 2 \mathrm{~cm}$.

It is immediately seen that now all components are smooth periodic functions, even those belonging to the removal cross section. However, the two components, having approximately the same magnitude, are out of phase (this follows 
directly from the different sign of the removal component in the noise source, as seen from (5) and (6)), and moreover they decay slightly differently in frequency. At the frequency where their amplitude becomes equal, a full destructive interference takes place. And since in the total noise the component belonging to the removal cross section is dominating, this leads to the deep sink seen in the total noise.

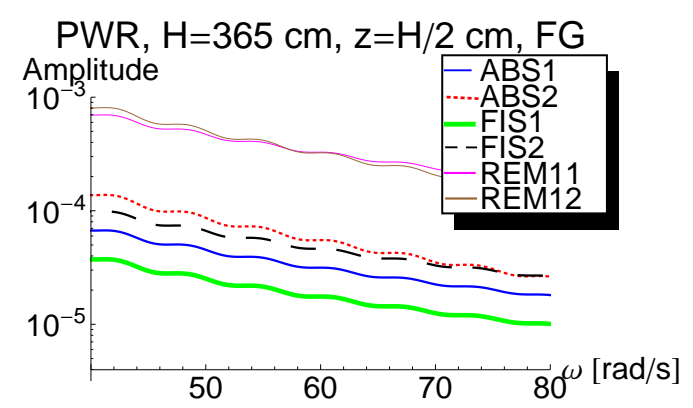

PWR, $\mathrm{H}=365 \mathrm{~cm}, \mathrm{z}=\mathrm{H} / 2 \mathrm{~cm}, \mathrm{TG}$

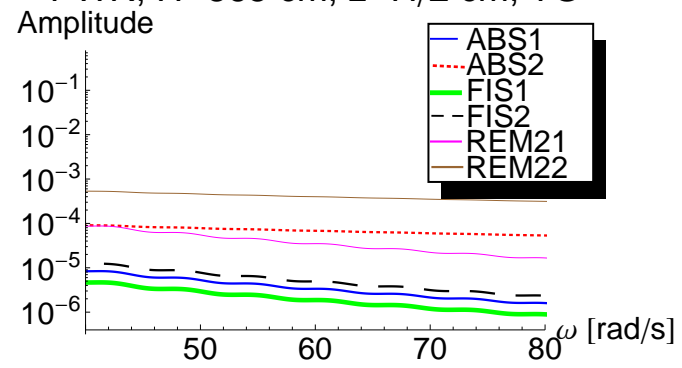

PWR, $\mathrm{H}=365 \mathrm{~cm}, \mathrm{z}=\mathrm{H} / 2 \mathrm{~cm}, \mathrm{TG}$

Phase

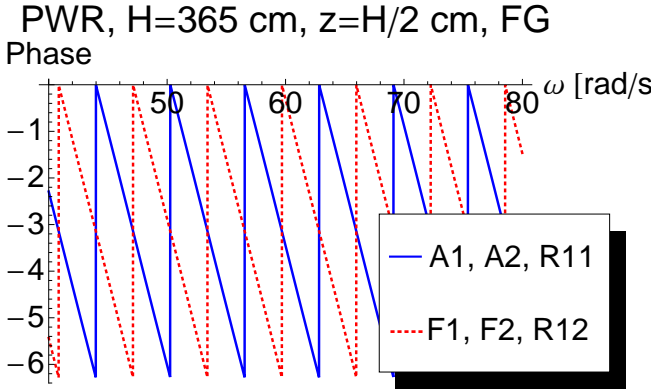

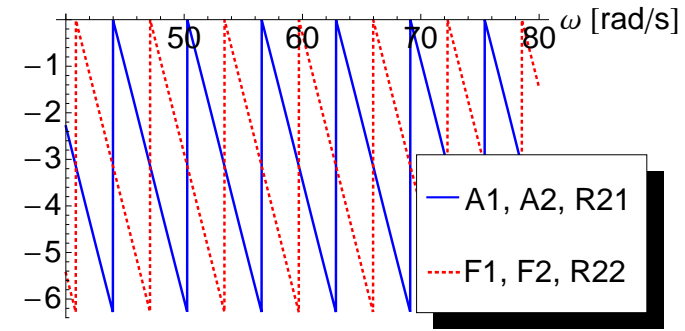

Fig. 13. Frequency dependence of the amplitude and phase of the neutron noise induced by propagating temperature perturbation in ABS1, ABS2, FIS1, FIS2, REM separately for a PWR in fast (left figures) and thermal (right figures) groups, respectively for $z=H / 2 \mathrm{~cm}$.

The different slope of the two components of the removal noise is, on the other hand related to the different weight of the local component in the two Green's functions. This is illustrated in Fig. 14 which shows the frequency dependence of the amplitude and the phase of the local and global components on the fast noise, contained in the Green's matrix elements $G_{11}$ and $G_{12}$, respectively. It is seen that the global components of the neutron noise corresponding to the $G_{11}$ and $G_{12}$ components exhibit a similar structure and are almost equal in their amplitudes. At the same time, as already argued for it earlier, the frequency decay of the local component of the noise is very much slower than that of the global component, in addition of being also a smooth function in frequency. In addition, as the figure also shows, the local component of the noise is much larger in the term due to $G_{12}$ than in that due to $G_{11}$. Further, in the term due to $G_{12}$, the local component is out-of-phase with the global component. These facts together lead to the different slope of $G_{11}$ and $G_{12}$ such that at a certain frequency their magnitude becomes equal. Since the noise induced by the fluctuation of the removal cross section is due to $G_{11}-G_{12}$, this leads to 

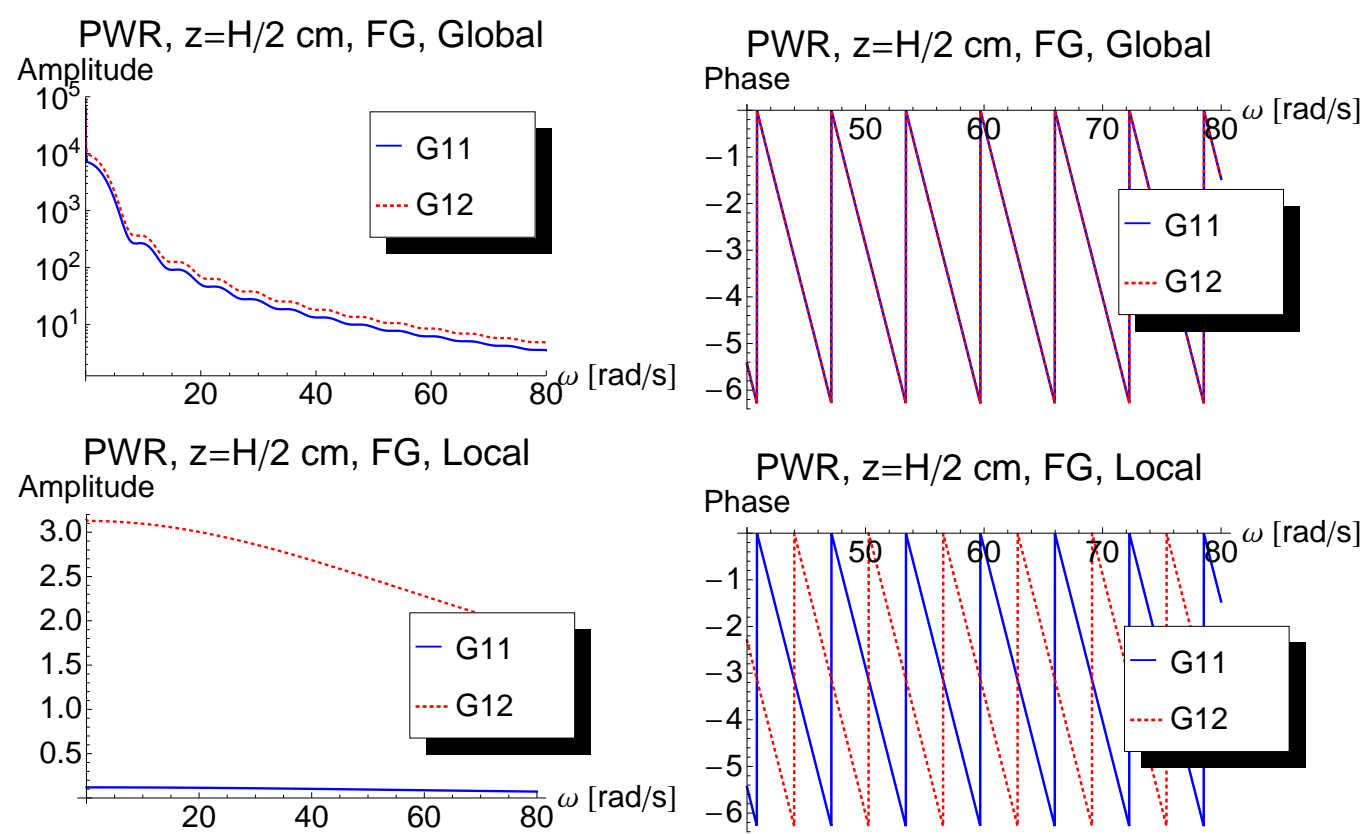

PWR, $z=H / 2 \mathrm{~cm}, F G$, Local

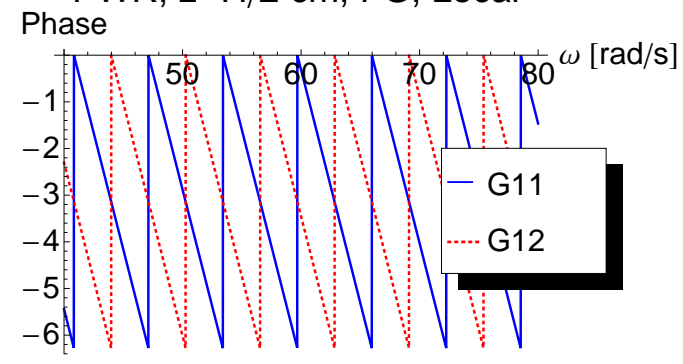

Amplitude

PWR, $z=H / 2 \mathrm{~cm}, F G$, Sum
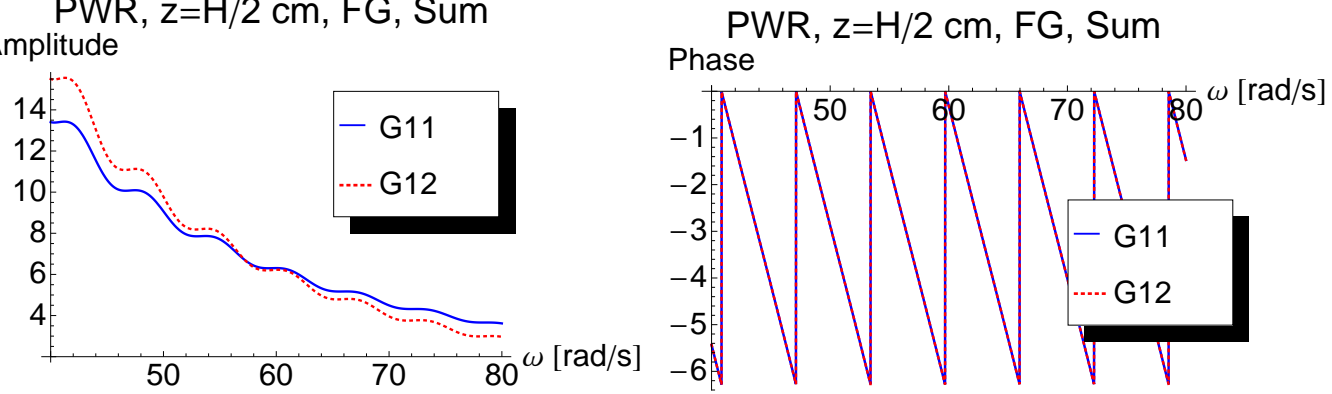

Fig. 14. Frequency dependence of the amplitude and the phase of the neutron noise induced by propagating temperature perturbation for a PWR for the global and local components and their sum in the fast group, respectively, for $z=H / 2 \mathrm{~cm}$.

the destructive interference, resulting in the dip in the fast noise around 50 $\mathrm{rad} / \mathrm{s}$ as shown in Fig. 9.

With a similar analysis, it can be shown that there is no similar effect in the thermal propagation noise in the PWR. It is seen in Fig. 11 that there is no deep dip in any of the noise components, including the one corresponding to the removal cross section. The main reason is that in the noise due to the removal cross section and $G_{22}$, the local and the global components are in phase. Hence the slopes of the two components $G_{21}$ and $G_{22}$ are diverging, and do not become comparable at any frequency. With a similar analysis all features of the spectra in Fig. 9 can be explained, i.e. why the dip frequency is different for the different cores, and why the dip occurs in the thermal noise in the MOX system, whereas it appears in the fast noise in the other three systems.

The reason why the possibility of such a single deep sink was not observed 
for the case of power reactors, i.e. where the point kinetic behaviour does not dominate, depends mostly on the fact that in light water reactors it only occurs in the fast noise, which was not investigated so far. On the other hand it is seen from the study presented here that in cores with different parameters, such as the MOX core with a fast spectrum, the dip occurs in the thermal noise, and moreover at a relatively low frequency which is monitored by most core surveillance systems.

\section{Conclusions, further work}

The investigations presented in this paper show that most of the properties of the fast and thermal propagation noise, found in recent work regarding molten salt reactors Jonsson and Pázsit (2011) is also present in traditional reactors, i.e. they are not a consequence of the propagating fuel and hence the propagating delayed neutron precursors and the accompanying difference in the dynamics of an MSR and a traditional core. These concern the spectral ratio of the neutron noise, the depth of the dips in the fast and thermal noise, and the decay of the amplitudes with increasing frequency. The spatially asymmetric characteristics of the noise could not be reproduced, which is a difference between the traditional systems investigated here and the MSR-type cores in the above publication.

One outcome of the quantitative work is that in particular in fast core systems, for a given propagating perturbation, the neutron noise is substantially larger in the fast group than in the thermal group. This observation might have a bearing on the use of noise diagnostic methods in fast systems such as an SFR (Sodium-Cooled Fast Reactor). The results suggest that it is beneficial to include fast neutron detection methods in the noise diagnostic system in fast reactors.

The results presented in this paper also underline the fact that for describing perturbations which affect several cross sections simultaneously, it is essential that the fluctuations in all cross sections are taken into account. This is the case at least when none of the cross section fluctuations dominates over the others. In BWRs, it was sufficient to consider only the fluctuations of the removal cross sections in order to have an in-depth understanding of the in-core noise. For the description of the noise related to temperature and density variations of one-phase low coolant, the present results suggest that it is necessary to model the noise source by accounting for its effect on all cross sections. This concern in particular molten salt reactors, in which propagating perturbations of various origin (temperature, density, fissile component etc.) are expected to occur. One can also add that the present findings add one more item to the list given in (Pázsit and Dykin, 2010) as to why the sink structure was never 
observed in the APSDs of in-core detectors in PWRs.

It has also to be mentioned that the propagation noise, as defined in the present paper, is not attributed to the noise induced by two-phase flow phenomena such as the boiling process in a BWR, rather by inhomogeneities of the one-phase coolant, such as fluctuations of the density and temperature. Such perturbations presumably too subtle in a PWR or a CANDU to be detected, and can be also suppressed by other noise sources and background noise. However, as it was mentioned in (Jonsson and Pázsit, 2011), such inhomogeneities are expected to be larger in an MSR, where the coolant is a mixture of fluoride salts, fuel, fission products, with local heat generation in the fuel dissolved in the coolant. Hence, it is expected that such effects will play a more significant role in an MSR. Whether or not they can be detected will depend on the in-core instrumentation in the design of the planned MSRs.

\section{Acknowledgement}

This work was financially supported by the Swedish Centre for Nuclear Technology and the Ringhals power plant.

\section{Appendix}

We start with the two-group diffusion equation written for the neutron noise in matrix form:

$$
\hat{\mathbf{L}}(\overline{\mathbf{r}}, \omega) \delta \overline{\mathbf{\Phi}}(\overline{\mathbf{r}}, \omega)=\overline{\mathbf{S}}(\overline{\mathbf{r}}, \omega)
$$

where

$$
\hat{\mathbf{L}}(\overline{\mathbf{r}}, \omega)=\left(\begin{array}{ll}
L_{11} & L_{12} \\
L_{21} & L_{22}
\end{array}\right)
$$

with

$$
\begin{gathered}
L_{11}=D_{1}(\overline{\mathbf{r}}) \nabla^{\mathbf{2}}-\boldsymbol{\Sigma}_{\mathbf{1}}(\overline{\mathbf{r}}, \omega), \\
L_{12}=\nu \Sigma_{f 2}(\overline{\mathbf{r}}, \omega), \\
L_{21}=\Sigma_{R}(\overline{\mathbf{r}}), \\
L_{22}=D_{2}(\overline{\mathbf{r}}) \nabla^{\mathbf{2}}-\boldsymbol{\Sigma}_{\mathbf{2}}(\overline{\mathbf{r}}, \omega), \\
\delta \overline{\boldsymbol{\Phi}}(\overline{\mathbf{r}}, \omega)=\left(\begin{array}{c}
\delta \phi_{1}(\overline{\mathbf{r}}, \omega) \\
\delta \phi_{2}(\overline{\mathbf{r}}, \omega)
\end{array}\right),
\end{gathered}
$$




$$
\overline{\mathbf{S}}(\overline{\mathbf{r}}, \omega)=\left(\begin{array}{c}
S_{1}(\overline{\mathbf{r}}, \omega) \\
S_{2}(\overline{\mathbf{r}}, \omega)
\end{array}\right) .
$$

The transposed form of Eq. (24) can be written as:

$$
[\hat{\mathbf{L}}(\overline{\mathbf{r}}, \omega) \delta \overline{\mathbf{\Phi}}(\overline{\mathbf{r}}, \omega)]^{\mathbf{T}}=[\overline{\mathbf{S}}(\overline{\mathbf{r}}, \omega)]^{\mathbf{T}} .
$$

After some rearrangements, one gets:

$$
\delta \overline{\boldsymbol{\Phi}}^{\mathbf{T}}(\overline{\mathbf{r}}, \omega) \hat{\mathbf{L}}^{\mathbf{T}}(\overline{\mathbf{r}}, \omega)=\overline{\mathbf{S}}^{\mathbf{T}}(\overline{\mathbf{r}}, \omega) .
$$

Multiplying both sides of Eq. (33) from the left-hand side by adjoint Greens function $\hat{\mathbf{G}}^{\dagger}\left(\overline{\mathbf{r}}, \overline{\mathbf{r}}^{\prime}, \omega\right)$ defined as:

$$
\hat{\mathbf{L}}^{\dagger}(\overline{\mathbf{r}}, \omega) \hat{\mathbf{G}}^{\dagger}\left(\overline{\mathbf{r}}, \overline{\mathbf{r}}^{\prime}, \omega\right)=\hat{\mathbf{I}} \cdot \delta\left(\overline{\mathbf{r}}-\overline{\mathbf{r}}^{\prime}\right)
$$

where $\hat{\mathbf{I}}$ is the $2 \times 2$ unit matrix and $\hat{\mathbf{G}}^{\dagger}\left(\overline{\mathbf{r}}, \overline{\mathbf{r}}^{\prime}, \omega\right)$ is given as:

$$
\hat{\mathbf{G}}^{\dagger}\left(\overline{\mathbf{r}}, \overline{\mathbf{r}}^{\prime}, \omega\right)=\left(\begin{array}{ll}
G_{11}^{\dagger} & G_{12}^{\dagger} \\
G_{21}^{\dagger} & G_{22}^{\dagger}
\end{array}\right)
$$

and

$$
\hat{\mathbf{L}}^{\dagger}=\hat{\mathbf{L}}^{\mathbf{T}}
$$

and integrating over the reactor core volume $V_{R}$, one gets:

$$
\int_{V_{R}} \delta \overline{\boldsymbol{\Phi}}^{\mathbf{T}}(\overline{\mathbf{r}}, \omega) \hat{\mathbf{L}}^{\mathbf{T}}(\overline{\mathbf{r}}, \omega) \hat{\mathbf{G}}^{\dagger}\left(\overline{\mathbf{r}}, \overline{\mathbf{r}}^{\prime}, \omega\right) \mathbf{d} \overline{\mathbf{r}}=\int_{\mathbf{V}_{\mathbf{R}}} \overline{\mathbf{S}}^{\mathbf{T}}(\overline{\mathbf{r}}, \omega) \hat{\mathbf{G}}^{\dagger}\left(\overline{\mathbf{r}}, \overline{\mathbf{r}}^{\prime}, \omega\right) \mathbf{d} \overline{\mathbf{r}}
$$

Combining Eqs. (34) and (36), the last equation can be rewritten as:

$$
\int_{V_{R}} \delta \overline{\mathbf{\Phi}}^{\mathbf{T}}(\overline{\mathbf{r}}, \omega) \hat{\mathbf{I}} \cdot \delta\left(\overline{\mathbf{r}}-\overline{\mathbf{r}}^{\prime}\right) \mathbf{d} \overline{\mathbf{r}}=\int_{\mathbf{V}_{\mathbf{R}}} \overline{\mathbf{S}}^{\mathbf{T}}(\overline{\mathbf{r}}, \omega) \hat{\mathbf{G}}^{\dagger}\left(\overline{\mathbf{r}}, \overline{\mathbf{r}}^{\prime}, \omega\right) \mathbf{d} \overline{\mathbf{r}}
$$

or in more compact form:

$$
\delta \overline{\boldsymbol{\Phi}}^{\mathbf{T}}\left(\overline{\mathbf{r}}^{\prime}, \omega\right)=\int_{\mathbf{V}_{\mathbf{R}}} \overline{\mathbf{S}}^{\mathbf{T}}(\overline{\mathbf{r}}, \omega) \hat{\mathbf{G}}^{\dagger}\left(\overline{\mathbf{r}}, \overline{\mathbf{r}}^{\prime}, \omega\right) \mathbf{d} \overline{\mathbf{r}}
$$

After changing of variables defined as:

$$
\overline{\mathbf{r}} \rightarrow \overline{\mathbf{r}}^{\prime}, \quad \overline{\mathbf{r}}^{\prime} \rightarrow \overline{\mathbf{r}} .
$$

Eq. (39) can be rewritten as:

$$
\delta \overline{\boldsymbol{\Phi}}^{\mathbf{T}}(\overline{\mathbf{r}}, \omega)=\int_{\mathbf{V}_{\mathbf{R}}} \overline{\mathbf{S}}^{\mathbf{T}}\left(\overline{\mathbf{r}}^{\prime}, \omega\right) \hat{\mathbf{G}}^{\dagger}\left(\overline{\mathbf{r}}^{\prime}, \overline{\mathbf{r}}, \omega\right) \mathbf{d} \overline{\mathbf{r}}^{\prime}
$$




\section{References}

Bell G. and Glasstone S. (1970), "Nuclear Reactor Theory", Van Nostrand Reinhold Company, New York.

Dam H. van. (1975), "A perturbation method for analysis of detector response to parametric fluctuations in reactors", Atomkernenergie 25, 70.

Dam H. van. (1976), "Neutron noise in boiling water reactors", Atomkernenergie 27, 8.

Dykin, V. and Pázsit, I. (2009), "Remark on the role of the driving force in BWR instability", Annals of Nuclear Energy 36, pp. 1544-1552.

Jonsson A. and Pázsit I. (2011), "Two-group theory of neutron noise in Molten Salt Reactors", Annals of Nuclear Energy 38, 6, pp. 1219-1246.

Kosály G. (1980), "Noise investigations in boiling-water and pressurized-water reactors", Progress in Nuclear Energy, 5, pp. 145-199.

Pázsit I. and Demazière C. (2010), "Noise Techniques in Nuclear Systems", Chapter in Handbook of Nuclear Engineering-Reactors of Generation II, Ed. D. G. Cacuci, Vol. 3, Chapter 2, Springer Verlag, New York, USA.

Pázsit I. (2002), "Neutron noise theory in the $P_{1}$ approximation", Progress in Nuclear Energy 40, pp. 217-236.

Pázsit I. and Dykin V. (2010), "Investigation of the space-dependent noise induced by propagating perturbations", Annals of Nuclear Energy 37, pp. 217-236.

Pázsit I. and Jonsson A. (2010), "Reactor kinetics, dynamic response and neutron noise in Molten Salt Reactors (MSR)", Nuclear Science and Engineering 167, pp. 61-76.

Williams M.M.R. (1974), "Random Processes in Nuclear Reactors", Pergamon Press, Oxford, New York, Tokyo.

Yoshioka H., Pázsit I., Otake H. and Nishina K. (1998) "Analysis of temporal and spatial decay of pulsed neutrons in single- and multiple-core systems", Annals of Nuclear Energy 25, pp. 1169 - 1193.

Studsvik Scandpower (2001), "SIMULATE-3: Andvanced Three-Dimensional Two-Group Reactor Analysis Code" , The User's Manual for SIMULATE-3, Studsvik Scandpower Inc., USA. 